\title{
Análisis participativo de servicios ecosistémicos en un área protegida del bosque seco tropical (bs-T), Colombia
}

\author{
Participatory analysis of ecosystem services in a protected area of \\ the tropical dry forest (TDF), Colombia
}

\author{
Luis Mario Cárdenas-Camacho ${ }^{1 *}{ }^{(\mathbb{D}}$, Sebastián Díaz $^{10}$, Wilson Gómez-Anaya $^{(\mathbb{D})}$, \\ John Eduard Rojas-Rojas ${ }^{2}$, René López-Camacho ${ }^{1}$
}

Cárdenas-Camacho, L. M., Díaz, S., Gómez-Anaya, W., Rojas-Rojas J. E. y López-Camacho, R. (2021). Análisis participativo de servicios ecosistémicos en un área protegida del bosque seco tropical (bs-T), Colombia. Colombia Forestal, 24(1), 123-156.

Recepción: 18 de junio 2020

\section{Resumen}

El estado de degradación que enfrentan los ecosistemas de bosque seco tropical (bs-T) en diferentes escalas geográficas es razón para priorizar su conservación. En este estudio se hizo un análisis participativo y multicriterio de servicios ecosistémicos (SE) asociados a coberturas presentes en la zona de vida de bs-T en un área protegida. Los SE fueron espacializados a una escala local para proyectar pautas de manejo, los cuales se priorizaron y caracterizaron mediante entrevistas a actores clave. Para la aproximación al funcionamiento del ecosistema se establecieron indicadores de procesos y estructura ecosistémica basados en información de suelos, vegetación, fauna e impulsores de cambio. Se encontró que los SE de mayor importancia son la producción agropecuaria y el suministro de agua potable. El funcionamiento del ecosistema resultó bajo y muy bajo en sistemas agrícolas; bajo y medio en sistemas pecuarios; y alto y muy alto en sistemas naturales. Se
Aprobación: 21 de septiembre 2020

recomienda poner en funcionamiento estrategias de conservación sostenibles y participativas.

Palabras clave: análisis participativo, multi-criterio, planeación del paisaje, manejo forestal sostenible.

\begin{abstract}
The state of degradation faced by tropical dry forest ecosystems, in different geographical scales, prioritizes their conservation. In this study, a participatory and multicriteria analysis of ecosystem services -ESassociated with current coverages in the tropical dry forest life zone in a protected area, was realized. The ES were spatialized on a local scale, to project management guidelines, and were prioritized and characterized through interviews with key stakeholders. For the approach to the ecosystem functioning, indicators of processes and ecosystem structure were established based on information of soils, flora, fauna and drivers of change. It was found that the most important ES are agricultural production and
\end{abstract}

1 Universidad Distrital Francisco José de Caldas, Facultad del Medio Ambiente y Recursos Naturales. Bogotá, Colombia.

2 Corporación Autónoma regional de Cundinamarca, Dirección de Recursos Naturales. Bogotá, Colombia.

Imariocc@gmail.com. Autor de correspondencia. 
drinking water supply. The ecosystem functioning was low and very low in agricultural systems, low and medium in livestock systems, and high and very high in natural systems. It is recommended to implement sustainable and participatory conservation strategies.

Keywords: participatory analysis, multi-criteria, landscape planning, sustainable forest management.

\section{INTRODUCCIÓN}

El bosque seco tropical (bs-T) es uno de los ecosistemas más amenazados en el mundo. Cerca del $48.5 \%$ de su área ha sido transformada (Hoekstra et al., 2004) y se considera como prioridad para la conservación, restauración y manejo (Trejo y Dirzo, 2000). Así mismo, estos bosques proveen una variedad de servicios ecosistémicos $(\mathrm{SE})$, principalmente de suministro de alimento y productos forestales no maderables (Balvanera et al., 2011); e importantes servicios de regulación asociados al secuestro de carbono, control de erosión, mantenimiento de la fertilidad del suelo, calidad de agua y bellezas escénicas (Maass et al., 2005). A pesar de esto, se estima que entre el $10 \%$ y el $20 \%$ del bs-T está en proceso de degradación y cerca del $30 \%$ enfrenta amenazas ante escenarios de cambio climático, cambio de uso de suelo y crecimiento poblacional que afectan su funcionamiento, reducen la resiliencia del ecosistema y vulneran a las comunidades humanas que allí habitan, al comprometer su seguridad alimenticia (Yirdaw et al., 2017).

En Colombia se identifican seis regiones de bs-T ( $8 \%$ del ecosistema original), siendo el valle del Magdalena una de las que presenta mayores amenazas (González-M. et al. 2018). Específicamente, para el bs-T del departamento de Cundinamarca, García-Márquez et al. (2016) indican la urgencia por concretar esfuerzos de conservación en este ecosistema. En suma, es evidente la presencia de vacíos de conocimiento sobre la cuantificación y tendencias de SE, en particular para los asociados con el agua y el suelo (Calvo-Rodríguez et al., 2016).
En este contexto, la Corporación Autónoma Regional de Cundinamarca (CAR) definió el Distrito regional de manejo integrado (DMI) Bosque Seco de la Vertiente Oriental del Rio Magdalena, mediante el Acuerdo 20 del 17 de julio de 2018 (CAR, 2018). Esto con el fin de crear una figura de área protegida que contribuya a la conservación de la biodiversidad y los SE, así como mantener la capacidad productiva del ecosistema y las condiciones ecológicas (art. 3). Del mismo modo, se resalta la construcción participativa con los actores del área para el mantenimiento de los objetos de conservación (art. 4).

Apoyado en la iniciativa mencionada, el presente estudio tuvo como objeto hacer una aproximación local al análisis participativo de los SE asociados a las diferentes coberturas presentes en el área del DRMI, cuya clasificación climática corresponde a la zona de vida bs-T, acorde a la clasificación de Holdridge (1967). Esto con el fin de brindar a las autoridades ambientales y territoriales una descripción práctica del actual funcionamiento de este ecosistema estratégico para así generar pautas para la toma de decisiones sobre el manejo de esta área.

\section{MATERIALES Y MÉTODOS}

\section{Área de estudio}

El área de estudio corresponde el DRMI bosque seco de la vertiente oriental del rio Magdalena, definido por la CAR (2018) en las provincias centro y alto Magdalena; teniendo en cuenta que estas presentan el menor estado de fragmentación y la 
mayor extensión de cobertura boscosa. Este espacio comprende la franja de bs-T de la vertiente media oriental del río Magdalena en el departamento de Cundinamarca, incluyendo los municipios de Nariño, Guataquí, Jerusalén, Beltrán, Pulí y San Juan de Rioseco (figura 1). Así mismo, posee una extensión de 36126.93 ha y su objetivo de manejo es garantizar la oferta de bienes y servicios ambientales esenciales para el bienestar humano correspondientes a fauna, flora, paisajes y recurso hídrico que ofrece este ecosistema.

La actividad socioeconómica del área se encuentra ampliamente relacionada con los SE de aprovisionamiento. Esto se debe a que el principal sector económico de la provincia del Magdalena centro, que coincide geográficamente con parte de los municipios asociados al DRMI (San Juan de Ríoseco, Beltrán y Pulí), es el de agricultura, ganadería, caza, silvicultura y pesca, con un aporte del $34.4 \%$ al PIB provincial (Gobernación de Cundinamarca, 2020). Además, la mayoría de la población rural de las subcuencas de la vertiente oriental del río Magdalena (Nariño, San Juan de Ríoseco y Beltrán) es de origen campesino y se dedica a la producción agrícola en minifundios o en alquiler (CAR, 2006).

Teniendo en cuenta la variedad de los sistemas naturales y agropecuarios, el área de estudio se dividió en tres grandes tipos de ecosistemas, de acuerdo con lo establecido en el mapa de ecosistemas de Colombia (Instituto de Hidrología, Meteorología y Estudios Ambientales y Ministerio a Ambiente y Desarrollo Sostenible, 2017) y se definieron las siguientes unidades de análisis: I) ecosistemas naturales, ocupan un $59 \%$ del área total y agrupa las coberturas boscosas $(10.23 \%)$ y arbustivas densas y abiertas (48.92\%); II) agroecosistemas agrícolas, ocupan el $5 \%$ del área total y agrupa las coberturas de cultivos permanentes, anuales, transitorios, arbóreos, arbustivos y herbáceos y los mosaicos de cultivos; y III) agroecosistemas pecuarios, con una extensión del $33 \%$ del área total, que agrupa los pastos limpios, enmalezados y arbolados.

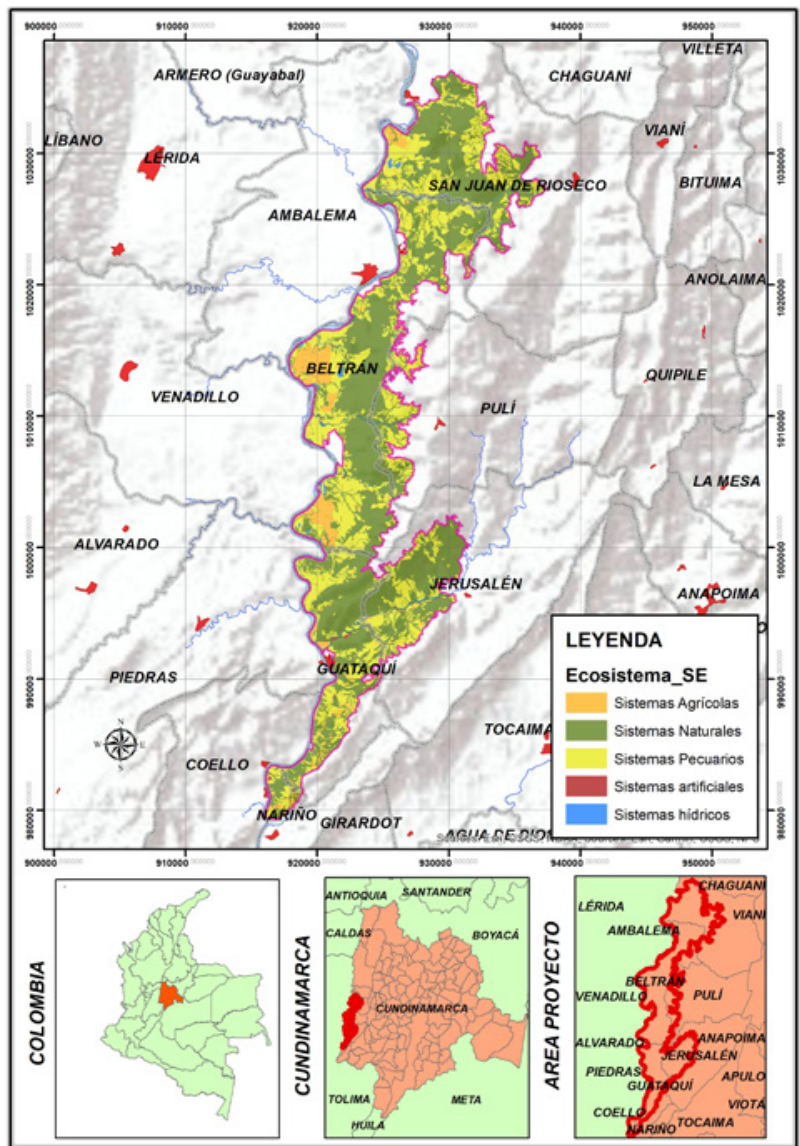

Figura 1. Área de estudio de bs-T del departamento de Cundinamarca.

\section{METODOLOGÍA}

Se siguió la metodología expuesta por Bubb et al. (2017) para la evaluación de los SE, que presenta un marco para el fortalecimiento de la planificación a diferentes escalas de gestión; a través de la inclusión de los SE y su relación con el funcionamiento del ecosistema. Este esquema prevé seis pasos, descritos a continuación:

\section{Paso 1: definición del contexto del área de estudio y el grupo de beneficiarios de los SE asociados al bs-T}

A partir de recorridos de campo se verificó y validó la información cartográfica a escala 1:25 000. 
Con lo anterior, se identificaron y confirmaron los principales usos actuales del suelo. Cabe destacar que dentro del área protegida se reconocen 1047 predios, de los cuales el $50.4 \%$ son inferiores a 5 ha y el $27.0 \%$ se encuentra entre 10 y 50 ha; $y$ cuya principal actividad productiva es agropecuaria (CAR, 2017).

\section{Pasos 2 y 3: identificación de la demanda y suministro actual de SE}

Se realizaron 11 entrevistas semiestructuradas a actores claves como líderes campesinos, presidentes de juntas de acción comunal y funcionarios públicos (véase el anexo suplementario). En general, se buscaba conocer la percepción de los actores frente a tres SE, con 24 preguntas asociadas a la importancia y uso (Fagerholm et al., 2012). Además, se consultó información oficial reportada por los municipios en lo referente al tema de abastecimiento de agua y producción agrícola y pecuaria (Gobernación de Cundinamarca, 2016; Contraloría de Cundinamarca, 2016). Los SE identificados se clasificaron en las categorías propuestas por Bubb et al. (2017).

\section{Paso 4: evaluación del funcionamiento actual de algunos procesos ecosistémicos}

Se identificó la cartografía base e imágenes satelitales rapideye, usando datos suministrados por el Instituto Geográfico Agustín Codazzi (Igac) (2017). Con base en esta información se elaboró la cartografía temática a escala 1:25 000 para geomorfología, suelos, coberturas, ecosistemas, unidades vegetales y unidades tróficas; estas dos últimas soportadas en los levantamientos de vegetación y fauna silvestre. De acuerdo con lo anterior, se definieron indicadores para evaluar los procesos ecosistémicos y la estructura del ecosistema en cada unidad de análisis (figura 2); la valoración para cada indicador se presenta en la tabla 1. Específicamente, los suelos se caracterizaron a partir de 28 perfiles modales representativos de las unidades de paisaje del área de estudio y 65 muestras de horizontes de suelo, de acuerdo con la metodología del Igac (2010). La vegetación se caracterizó a partir de 18 parcelas de 0.1 ha $(50 \mathrm{~m} \times 20 \mathrm{~m})$, según lo establecido por la red DryFlor (Moonlight et al., 2020), y 84 puntos de recolección libre de material botánico.

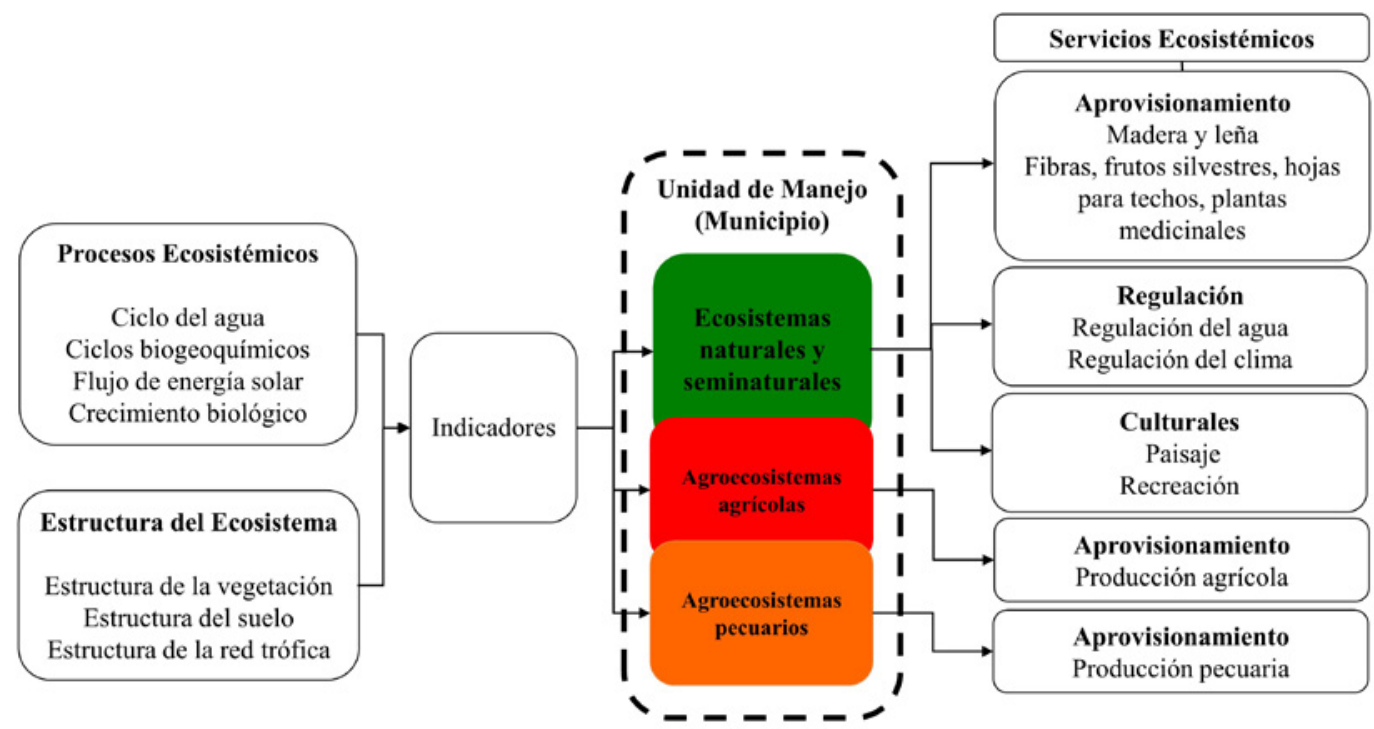

Figura 2. Esquema metodológico usado en la planificación de la gestión de los SE en el área de bs-T del departamento de Cundinamarca con base en Bubb et al. (2017). 
En paralelo, se evaluó la abundancia de los siguientes grupos: insectos (Coleóptera y Lepidóptera), siguiendo lo establecido por Villareal et al. (2004); anfibios y reptiles, acorde a Crump y Scott (2001); aves, en función de la propuesta de Ralph et al. (1996); y mamíferos, según Tirira (2007) y Reid (2009).

Posteriormente, se realizó un análisis multicriterio mediante un método cuantitativo basado en un modelo de ponderación lineal denominado Scoring (Mendoza y Macoun, 1999), el cual se desarrolló en un ambiente SIG en la plataforma ArcGIS (Environmental Systems Research Institute, 2012) mediante el uso de las herramientas Model Builder y Weighted Overlay; lo que permitió ponderar los criterios e indicadores de acuerdo con la valoración asignada (tabla 1). Una vez obtenidos los resultados, se determinó la condición o estado de los SE a nivel espacial para el área de estudio. Adicionalmente, se realizó un análisis de componentes principales (ACP) (Wold et al., 1987) con el fin de identificar tendencias de aporte de las unidades administrativas (municipios) y de las unidades de análisis en el funcionamiento de los procesos ecológicos y la estructura de los ecosistemas. También se empleó un análisis de clúster jerárquico mediante el método de Ward (1963) para la delimitación de grupos utilizando las librerías FactoMineR (Le et al., 2008) y Factoextra (Kassambara y Mundt, 2018) del software R (R Development Core Team, 2017).

\section{Paso 5: identificación de los impulsores directos de cambio generalmente asociados a actividades humanas en lo local y regional}

Se realizó mediante la observación de 84 puntos en campo, entrevistas a actores clave de las comunidades locales y funcionarios municipales. Los impulsores se categorizaron según su nivel de impacto a partir de la clasificación de González et al. (2018) para ecosistemas naturales y para ecosistemas agrícolas y pecuarios. Adicionalmente, se asociaron estos impulsores de cambio con causas subyacentes o indirectas, acorde a lo propuesto por el Millennium Ecosystem Assessment Panel (2005).

\section{Paso 6: establecimiento de recomendaciones de manejo para la conservación y uso sostenible del bs-T asociada al marco normativo e institucional vigente}

Teniendo en cuenta que el artículo 14 del Decreto 2372 de 2010 (Ministerio de Ambiente, Vivienda y Desarrollo Territorial, 2010) define los DMI como espacios geográficos que mantienen la composición y función ecosistémica, a pesar de la modificación estructural, se procedió a construir las recomendaciones de manejo. Así mismo, se consideraron los usos permitidos dentro del área protegida (uso sostenible, preservación, restauración, conocimiento y disfrute) (CAR, 2018).

\section{RESULTADOS}

Los resultados de las entrevistas realizadas a los actores indican que los SE que presentan mayor importancia son los de aprovisionamiento, los cuales son tangibles y están asociados a la generación de ingresos (como la producción agrícola y ganadera) y aquellos que soportan la supervivencia y productividad, como es el caso del suministro de agua. A los servicios de aprovisionamiento como la carne silvestre, la pesca, la leña y las plantas medicinales se les concedió una importancia media; en tanto que los SE que no son vitales para la supervivencia, como los materiales para artesanías, los colorantes y los elementos decorativos obtuvieron una importancia baja. Con respecto a la relevancia de la recuperación de sitios en los que ocurren procesos de remoción en masa, se mencionan principalmente las zonas con mayor pendiente. Como parte de los SE de tipo cultural, se observó que los pobladores identifican un gran potencial en la zona de estudio para actividades de recreación y descanso, relacionados con la belleza paisajística y los atractivos naturales de la biodiversidad (tabla 2). 


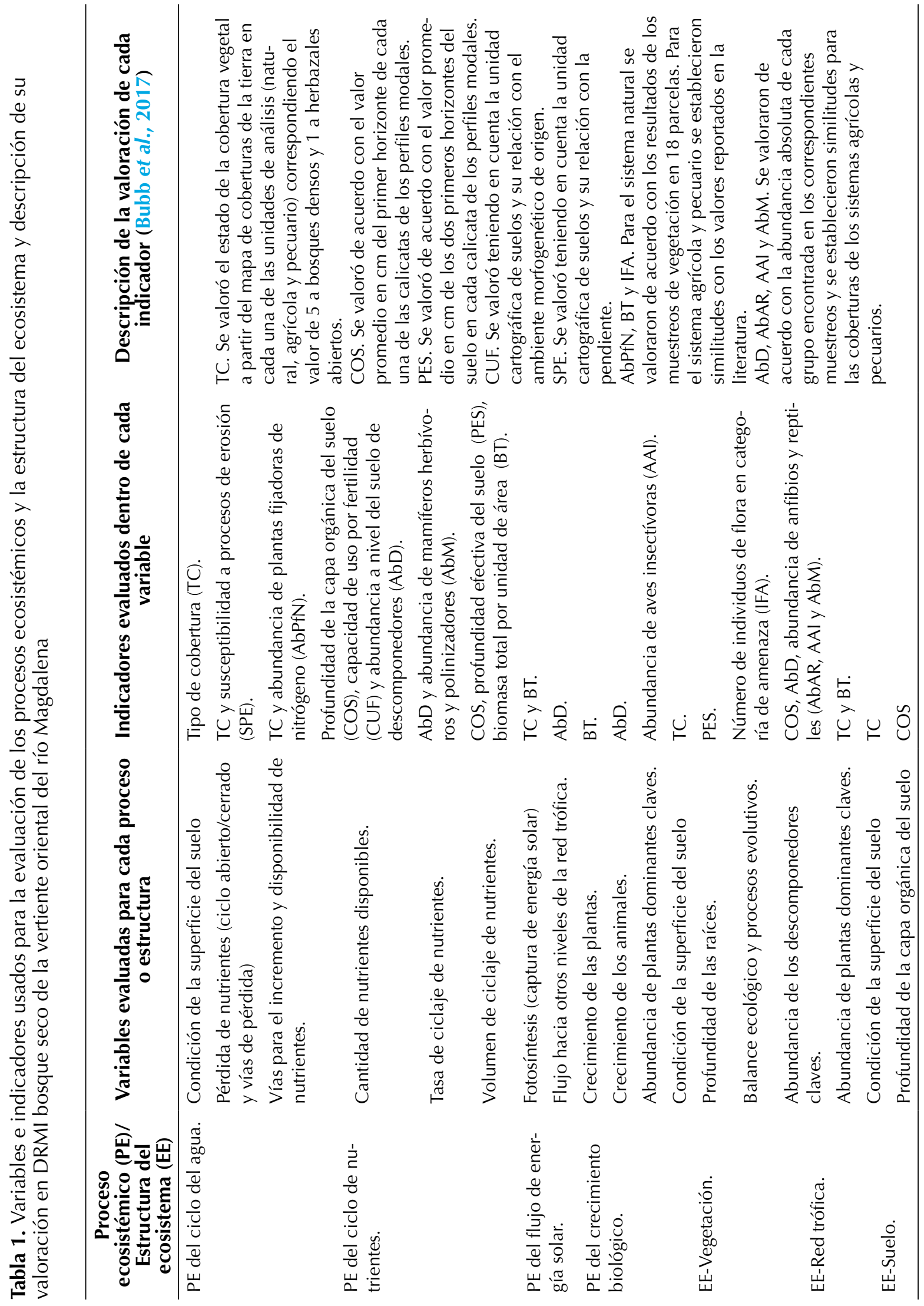


En cuanto a los resultados asociados al funcionamiento de los ecosistemas, a partir de los indicadores de procesos ecosistémicos y la estructura del ecosistema, es evidente la relación espacial de valores altos y muy altos con los ecosistemas naturales. Ello en tanto que los valores bajos y muy bajos se encuentran asociados a los sistemas agrícolas, en especial a los cultivos tecnificados de arroz, y en algunas zonas a los sistemas pecuarios (figura 3). Para los procesos ecosistémicos se encontró que las coberturas naturales y seminaturales presentan los mayores porcentajes de área en las categorías de alto y muy alto, los sistemas agrícolas presentan los mayores porcentajes de área en las categorías de bajo y muy bajo y los sistemas pecuarios se distribuyen en las categorías de bajo, medio y alto. Respecto a la estructura de los ecosistemas se halló la misma tendencia, con excepción de los sistemas agrícolas, que presentan los mayores porcentajes de área en la categoría de bajo (figura 4).

De manera complementaria, los análisis estadísticos reafirman los resultados encontrados en los análisis cartográficos. En los ACP se identificaron dos grupos diferenciados por su aporte en el funcionamiento de los procesos ecológicos y estructura de los ecosistemas, reuniendo el $61.4 \%$ y el $89.2 \%$ de la varianza de los datos, respectivamente (figura 5). El primero se caracterizó por presentar valores altos y muy altos en la calificación de su funcionamiento y en él se encontraron, en mayor proporción, las unidades correspondientes a los ecosistemas naturales de Beltrán, Guataquí, Jerusalén y San Juan de Rioseco. El segundo se caracterizó por estar asociado con valores bajos y muy bajos a los sistemas agrícolas y pecuarios presentes en todos los municipios.

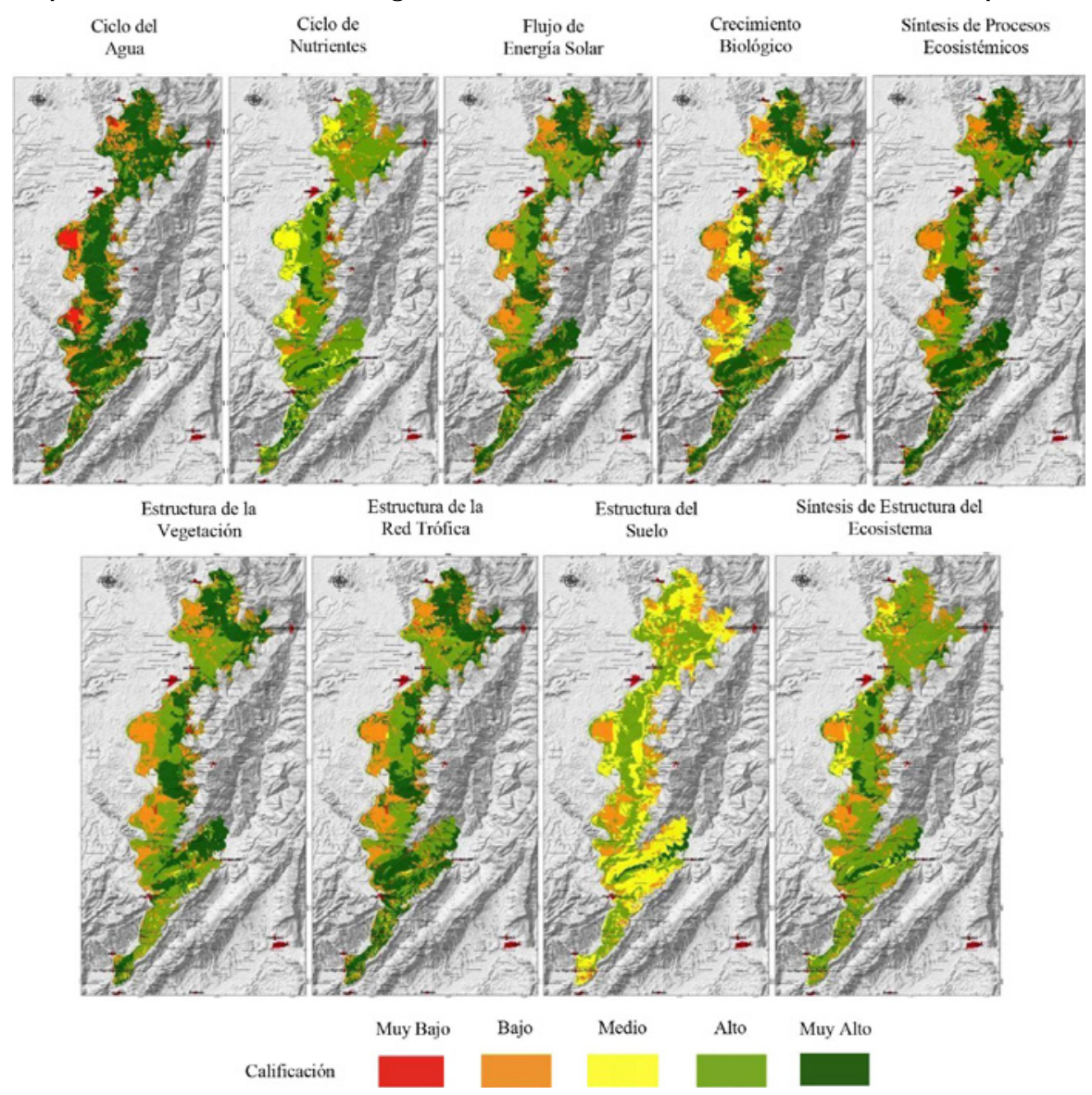

Figura 3. Mapas síntesis de los cuatro procesos ecosistémicos y los tres tipos de estructura del ecosistema evaluada para el área de bs-T del departamento de Cundinamarca. 


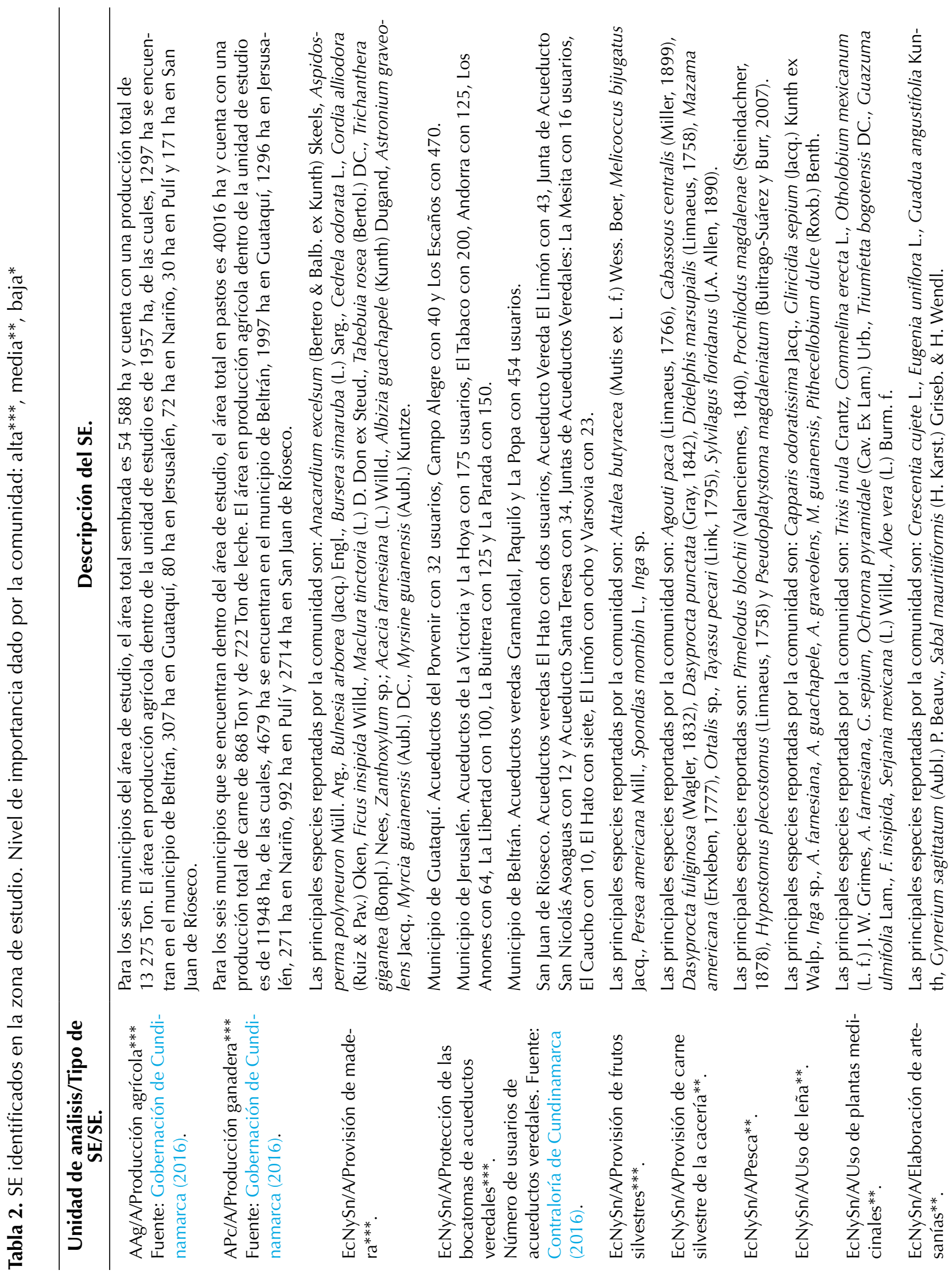




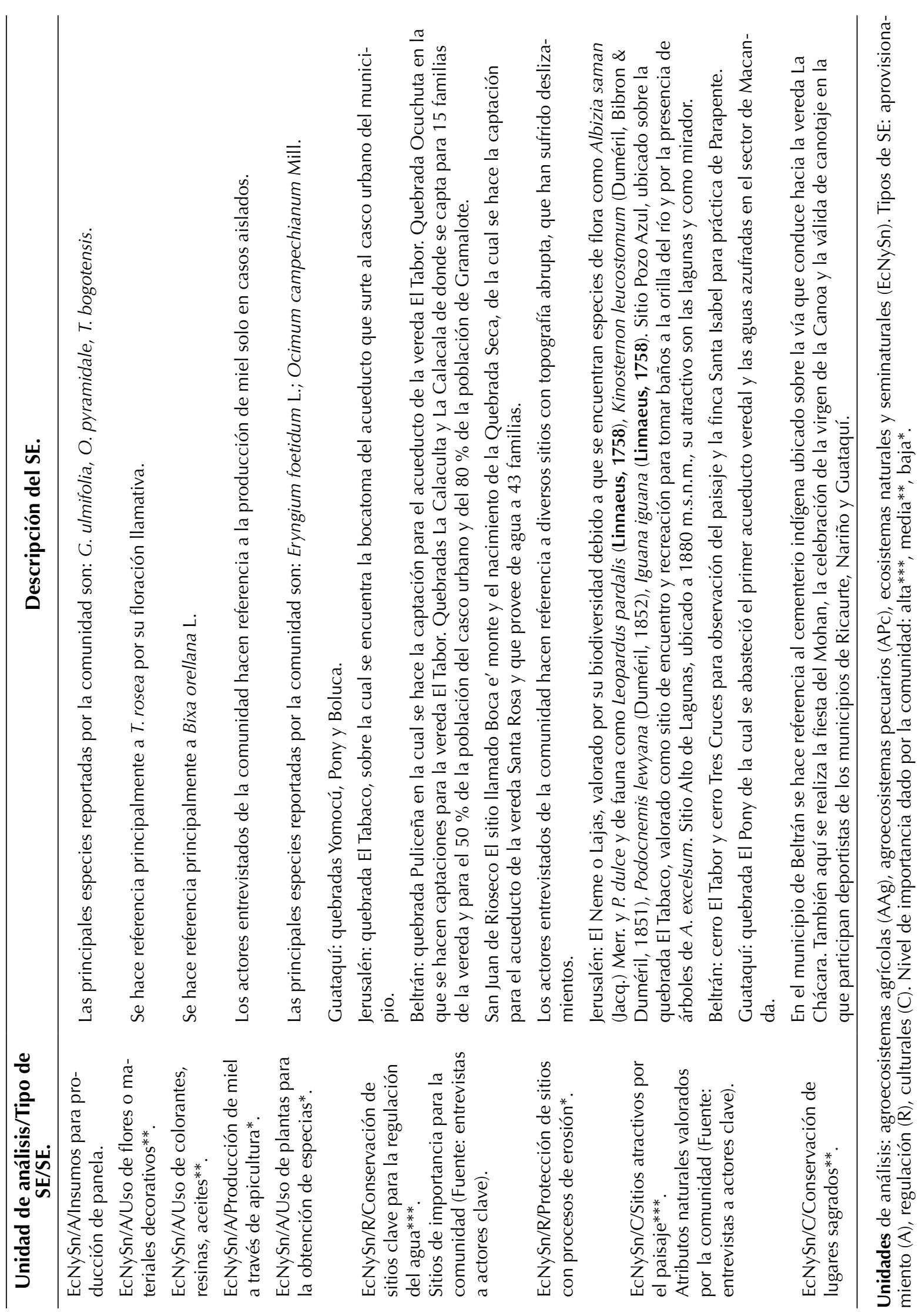




\begin{tabular}{|c|c|c|c|c|c|c|}
\hline & & Ciclo del agua Ciclo de nut & rientes & Flujo de energía & Crecimiento biológice & Procesos Ecosistémicos \\
\hline \multirow{5}{*}{ Sistemas Agricolas } & Muy bajo & 85,4 & 0,0 & 0,1 & 0 , & 6,7 \\
\hline & Bajo & 5,5 E & 10,7 & 86,3 & 91 , & 80,9 \\
\hline & Medio & 6,5 & 87,4 & 11,8 & 6, & 10,6 \\
\hline & Alto & 0,7 & 0,8 & 0,2 & $\mathbf{0}$, & 1,7 \\
\hline & Muy alto & 1,8 & 1,0 & 1,7 & 1, & 0,0 \\
\hline \multirow{5}{*}{$\begin{array}{c}\text { Sistemas Naturales y } \\
\text { Seminaturales }\end{array}$} & Muy bajo & 0,3 & 0,1 & 0,2 & 0 & 0,1 \\
\hline & Bajo & 0,2 & 0,3 & 0,3 & 0 , & 0,3 \\
\hline & Medio & 0,0 & 0,2 & 0,0 & 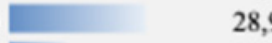 & 38,7 \\
\hline & Alto & 0,4 & 77,2 & 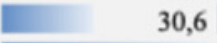 & E & 57,5 \\
\hline & Muy alto & 99,1 & 22,3 & 68,8 & 58, & 3,3 \\
\hline \multirow{5}{*}{ Sisitemas Pecuarios } & Muy bajo & 2,1 & 1,7 & 2,1 & 2 , & 0,2 \\
\hline & Bajo & $55,3 \square$ & 28,0 & 55,4 & 55 & 32,4 \\
\hline & Medio & 0,7 & 28,4 & 0,7 & 0 , & 50,5 \\
\hline & Alto & 38,8 & 41,6 & 39,0 & 41, & 16,8 \\
\hline & Muy alto & 3,0 & 0,2 & 2,8 & 0, & 0,0 \\
\hline & & \begin{tabular}{|l} 
Estructura de la vegetación \\
\end{tabular} & Estruct & tura red trófica & Estructura del suelo & Estructura del ecosistema \\
\hline \multirow{5}{*}{ Sistemas Agricolas } & Muy bajo & 0,1 & & 0,1 & 6,7 & 0,1 \\
\hline & Bajo & 90,7 & & 90,9 & 80,9 & 86,3 \\
\hline & Medio & 6,7 & & 6,6 & 10,6 & 11,1 \\
\hline & Alto & 1,6 & & 0,8 & 1,7 & 1,8 \\
\hline & Muy alto & 0,9 & & 1,7 & 0,0 & 0,8 \\
\hline \multirow{5}{*}{$\begin{array}{c}\text { Sistemas Naturales y } \\
\text { Seminaturales }\end{array}$} & Muy bajo & 0,3 & & 0,2 & 0,1 & 0,2 \\
\hline & Bajo & 0,2 & & 0,3 & 0,3 & 0,2 \\
\hline & Medio & 0,0 & & 0,1 & 38,7 & 0,2 \\
\hline & Alto & 46,1 & E & 37,7 & 57,5 & 79,0 \\
\hline & Muy alto & 53,4 & & 61,7 & 3,3 & 20,3 \\
\hline \multirow{5}{*}{ Sisitemas Pecuarios } & Muy bajo & 2,1 & & 2,1 & 0,2 & 2,1 \\
\hline & Bajo & 52,6 & & 55,3 & 32,4 & 37,2 \\
\hline & Medio & 3,5 & & 0,7 & 50,5 & 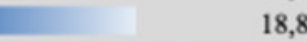 \\
\hline & Alto & 40,6 & & 39,1 & 16,8 & 41,1 \\
\hline & Muy alto & 1,2 & & 2,7 & 0,0 & 0,7 \\
\hline
\end{tabular}

Figura 4. Porcentaje de área acorde con la calificación asignada en el modelo en cada sistema evaluado para los principales procesos ecosistémicos (superior) y para los tipos de estructura del ecosistema (inferior).

En cuanto a los impulsores de cambio, se observó que las presiones actuales son el resultado de diferentes combinaciones entre impulsores directos y subyacentes de cambio. Los ecosistemas naturales rodeados generalmente de una matriz agrícola y ganadera tienen como mayor presión la presencia de ganado ( $44 \%$ de los puntos observados), la cercanía a infraestructura humana como carreteras, vivienda y zonas de extracción minera $(23 \%)$ y la tala selectiva (13\%); también, se menciona el impacto alto de quemas (6\%). Por otra parte, las actividades consideradas como de bajo impacto (como el ecoturismo y la extracción de productos forestales) son menos comunes en el área de estudio. En muchos casos, se evidencia una combinación de dos o tres causas próximas; frecuentemente, por ganadería (ramoneo) e infraestructura humana (bebederos, tuberías para extracción de agua, caminos). La tabla 3 sintetiza estos resultados y su nivel de impacto sobre el funcionamiento de los ecosistemas.

Por otro lado, el cambio climático, entendido como la alteración del régimen de lluvias y prolongación de la temporada seca, se identifica como el impulsor más importante para los sistemas agrícolas y pecuarios, seguido de los procesos de degradación y erosión del suelo que implican a pérdida de su capacidad productiva. También, la presencia de especies invasoras o con potencial invasor como Acacia farnesiana (L.) Willd (López-Camacho et al., 2012) se asocian generalmente a pastizales enmalezados y áreas degradadas; 

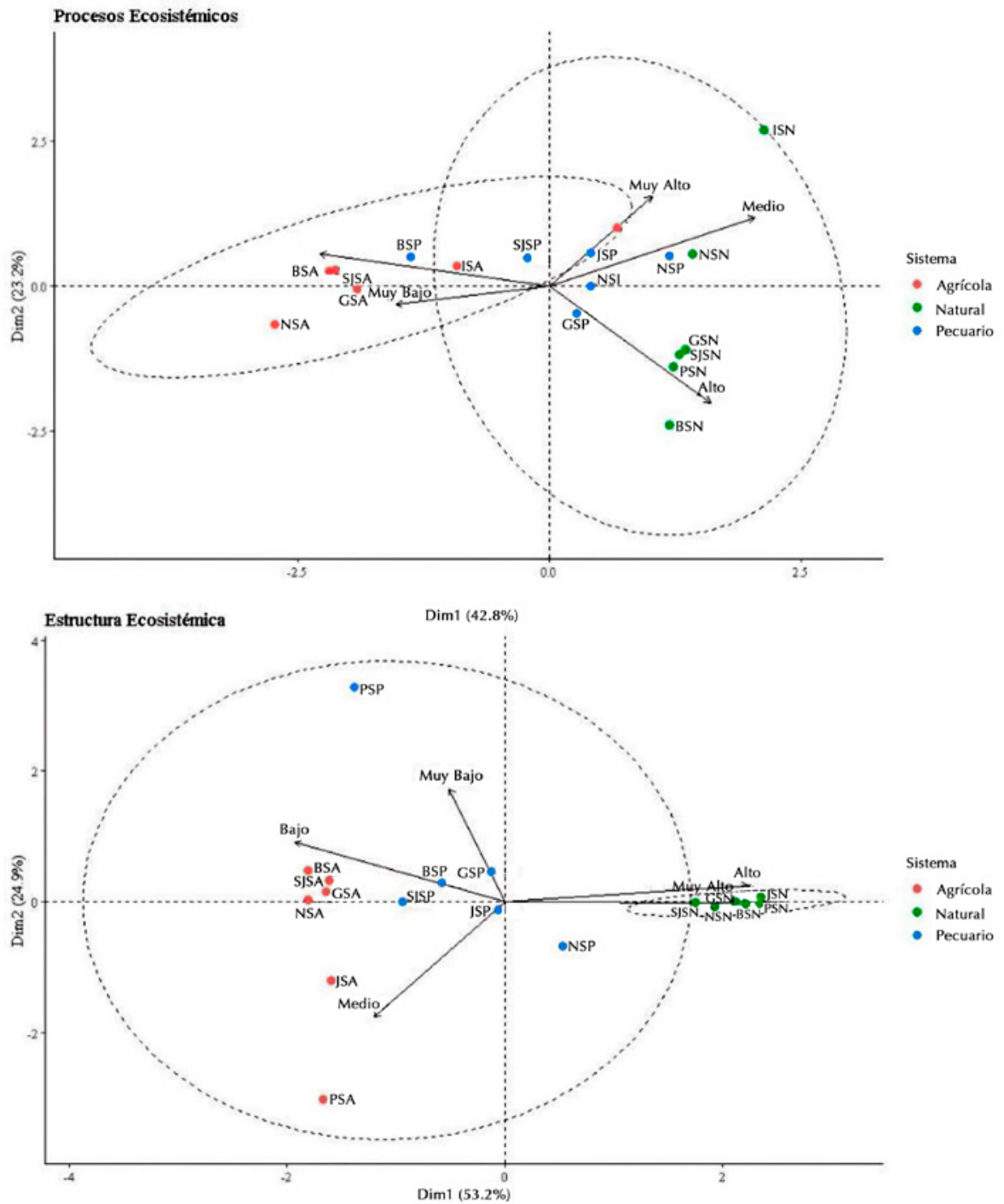

Figura 5. Tendencia del aporte de los diferentes municipios y ecosistemas a los procesos ecosistémicos (superior) y a la estructura del ecosistema (inferior) según el ACP. Las primeras iniciales de las unidades de aporte corresponden al municipio (B: Beltrán, G: Guataquí, J: Jerusalén; N: Nariño, P: Pulí, SJ: San Juan de Ríoseco) seguidas de los ecosistemas (SA: Agrícola, SP: Pecuario, SN: Natural).

y, según los habitantes de la zona, su erradicación genera costos elevados y la pérdida de terrenos para las actividades productivas. En suma, las fuerzas impulsoras subyacentes más importantes en los ecosistemas naturales son factores de tipo cultural y social, seguidas por factores económicos a pequeña escala asociados a cultivos transitorios y aprovechamiento de productos del bosque; mientras que, para los sistemas agrícolas y pecuarios, el crecimiento económico y los factores institucionales son las causas más importantes.

\section{Recomendaciones de manejo}

La implementación de acciones que incentiven la recuperación de los bosques secundarios mediante la sucesión permitirá mejorar la oferta de servicios cerca de las áreas agrícolas y pecuarias. 
Tabla 3. Impulsores de cambio directos asociados a los ecosistemas del área de estudio y sus implicaciones sobre el funcionamiento de los ecosistemas (impacto de la actividad: 1 muy bajo, 5 muy alto)

\begin{tabular}{|c|c|c|}
\hline Impulsores directos & Impacto probable sobre el funcionamiento del ecosistema & Impulsores indirectos \\
\hline \multicolumn{3}{|l|}{ Naturales. } \\
\hline $\begin{array}{l}\text { Ecoturismo, caza y ex- } \\
\text { tracción de productos no } \\
\text { madereros }(1) \text {. }\end{array}$ & $\begin{array}{l}\text { Estructura de la vegetación y red trófica. Pérdida de biodiversidad } \\
\text { y de procesos ecológicos dependientes de especies claves. }\end{array}$ & \multirow{5}{*}{$\begin{array}{l}\text { Factores culturales } \\
\text { religiosos y económicos. } \\
\text { Las actividades directas } \\
\text { sobre los ecosistemas } \\
\text { naturales generalmen- } \\
\text { te son prácticas de la } \\
\text { población rural para } \\
\text { acceder a materiales } \\
\text { para construcción, leña, } \\
\text { proteína u otros para su } \\
\text { uso directo o indirecto. }\end{array}$} \\
\hline Extracción selectiva (2). & Ibídem. Disminución de biomasa. & \\
\hline $\begin{array}{l}\text { Pastoreo de ganado dentro } \\
\text { del bosque y tala intensiva } \\
\text { (3). }\end{array}$ & $\begin{array}{l}\text { Estructura de la vegetación, ciclos biogeoquímicos, crecimiento } \\
\text { biológico y red trófica. Alteración en la biota e interrupción de } \\
\text { procesos ecológicos dependientes de especies clave. }\end{array}$ & \\
\hline Agricultura y ganadería (4). & $\begin{array}{l}\text { Ibídem. Pérdida de biodiversidad, procesos ecológicos y SE asocia- } \\
\text { dos a la regulación y soporte de hábitat principalmente. }\end{array}$ & \\
\hline $\begin{array}{l}\text { Infraestructura humana, } \\
\text { actividades mineras, hidro- } \\
\text { carburos y fuego (5). }\end{array}$ & $\begin{array}{l}\text { Estructura de la vegetación y ciclos biogeoquímicos. Cambios en } \\
\text { estructura y funcionamiento de ecosistemas. Afectación de ciclos } \\
\text { biogeoquímicos y pérdida de comunidad de descomponedores del } \\
\text { suelo. }\end{array}$ & \\
\hline \multicolumn{3}{|l|}{ Agrícolas. } \\
\hline $\begin{array}{l}\text { Infraestructura, actividades } \\
\text { mineras y fuego (1). }\end{array}$ & $\begin{array}{l}\text { Ciclos biogeoquímicos y cadenas tróficas. Altera la composición } \\
\text { del sistema. }\end{array}$ & \multirow{5}{*}{$\begin{array}{l}\text { Factores institucionales, } \\
\text { tecnológicos y cultura- } \\
\text { les. Diferencias entre } \\
\text { pequeños agricultores } \\
\text { que dependen de un } \\
\text { medio de vida rural y } \\
\text { grandes empresarios } \\
\text { con acceso a mercados } \\
\text { y tecnología. Falta de } \\
\text { opciones para reducir su } \\
\text { dependencia. }\end{array}$} \\
\hline $\begin{array}{l}\text { Contaminación por fertili- } \\
\text { zantes (2). }\end{array}$ & Ibídem, ciclo del agua. Cambio en la composición del suelo. & \\
\hline Especies invasoras (3). & $\begin{array}{l}\text { Estructura de la red trófica. Estructura y funcionamiento del siste- } \\
\text { ma. Agro-diversidad. }\end{array}$ & \\
\hline Cambio climático (4). & $\begin{array}{l}\text { Ciclo del agua y redes tróficas. Reducción y/o alteración de la } \\
\text { productividad y temporalidad de las cosechas. Mayor ocurrencia } \\
\text { de enfermedades y plagas. }\end{array}$ & \\
\hline $\begin{array}{l}\text { Degradación y erosión del } \\
\text { suelo (5). }\end{array}$ & $\begin{array}{l}\text { Ciclos biogeoquímicos, ciclo del agua y cadenas tróficas. Disminu- } \\
\text { ción de la fertilidad del suelo y evidencia de procesos de erosión. } \\
\text { Afectación de producción agrícola. }\end{array}$ & \\
\hline \multicolumn{3}{|l|}{ Pecuarios. } \\
\hline $\begin{array}{l}\text { Contaminación de fuentes } \\
\text { hídricas (1). }\end{array}$ & $\begin{array}{l}\text { Ciclos biogeoquímicos y alteración de red trófica. Generación de } \\
\text { enfermedades epidémicas y endémicas del ganado. Disminución } \\
\text { de la calidad del agua. }\end{array}$ & \multirow{5}{*}{$\begin{array}{l}\text { Factores económicos. } \\
\text { La ganadería contribuye } \\
\text { a los medios de vida de } \\
\text { la población generando } \\
\text { ingresos bien sea como } \\
\text { actividad principal o } \\
\text { como alternativa para } \\
\text { un ingreso adicional y } \\
\text { esporádico. }\end{array}$} \\
\hline $\begin{array}{l}\text { Degradación y erosión del } \\
\text { suelo (2). }\end{array}$ & $\begin{array}{l}\text { Ciclos biogeoquímicos y estructura del suelo. Agotamiento de } \\
\text { fertilidad del suelo y procesos de erosión en ladera. Afectación de } \\
\text { capacidad del suelo para retener y regular el agua. }\end{array}$ & \\
\hline Cambio climático (3). & $\begin{array}{l}\text { Ciclo del agua y redes tróficas. Aumento de la escasez de agua. } \\
\text { Reducción de la productividad. Mayor ocurrencia de enfermeda- } \\
\text { des y plagas. }\end{array}$ & \\
\hline Especies invasoras (4). & $\begin{array}{l}\text { Estructura de la vegetación y la red trófica. Afecta la composición } \\
\text { y estructura del sistema y la competencia entre especies. Origina } \\
\text { procesos de degradación de suelos. }\end{array}$ & \\
\hline $\begin{array}{l}\text { Sobreutilización del } \\
\text { suelo (5). }\end{array}$ & $\begin{array}{l}\text { Ciclos biogeoquímicos, red trófica. Agotamiento de la productivi- } \\
\text { dad del sistema pecuario. Disminución de plantas forrajeras. }\end{array}$ & \\
\hline
\end{tabular}


En este sentido, es urgente emprender acciones de regulación que eviten que en estas coberturas se presente conversión de uso del suelo para el establecimiento de pastos. Por lo que la implementación de mecanismos financieros como REDD+, los cuales buscan la captura de carbono, podrían establecerse para aumentar los beneficios de la biodiversidad derivados de la deforestación evitada (Phelps et al., 2012), contribuyendo al manejo de la vegetación y proporcionando un ingreso familiar alternativo.

Igualmente, los procesos ecosistémicos evaluados y los tipos de estructura del ecosistema para las zonas agrícolas y pecuarias muestran alteraciones en los procesos de ciclo del agua, flujo de energía, crecimiento biológico, estructura de la vegetación y estructura del suelo. Este resultado concuerda con lo reportado en el bosque seco del departamento del Tolima, en el cual algunos de los productores expresaron que los cambios a los que el ecosistema se ha visto sometido han tenido consecuencias negativas como el incremento de la temperatura media, el aumento de plagas, la desertificación y degradación del suelo, la disminución de la oferta de agua y la baja producción de arroz (Andrade et al., 2017). Adicionalmente, se potencian otros efectos de actividades productivas, como la ganadería, que contribuyen al establecimiento de especies invasoras y a los procesos de degradación del ecosistema al favorecer el establecimiento de arbustales mono específicos que a largo plazo modifican las propiedades del suelo (Vásquez-Valderrama et al., 2017, 2020).

El anterior panorama es evidencia de las decisiones de los pobladores locales sobre el uso del suelo, las cuales están relacionadas con su supervivencia personal y afectan directamente la provisión de SE a nivel individual y comunitario. Por lo cual, es necesario reconocer la relevancia de estas acciones locales tanto en las prioridades de investigación como en las políticas regionales y nacionales para evitar así una de las principales causas de la reducción en el suministro de SE (Paudyal et al., 2015).
De esta manera, se configura un escenario de alerta en el que se deben promover cambios inmediatos en los procesos de producción agrícola y pecuaria ya que, como lo afirman García et al. (2014), la probabilidad de mantener sistemas productivos bajo coberturas de áreas en desertificación y sobreexplotación es muy baja y acarrea diversos problemas sociales y económicos. A pesar de que Colombia cuenta con una Política Nacional de Gestión de la Biodiversidad y los Servicios Ecosistémicos y que las iniciativas de conocimiento y conservación del bs-T se han incrementado en los últimos años (Pizano y García, 2014), son aisladas las acciones que se realizan a nivel regional y local. Por lo tanto, deben proponerse estrategias de manejo que especifiquen una ruta metodológica sistemática en torno a un objetivo de conservación y uso e incorporen aspectos apropiados como la adopción de un enfoque de planificación participativa que considere las dinámicas ecológicas y la evaluación de los SE.

En síntesis, para avanzar hacia la conservación del bs-T es necesario fortalecer el interés común sobre la importancia de la provisión de SE, de tal manera que la formulación de políticas públicas y la investigación consideren de manera adecuada las acciones de los actores locales (Paudyal et al., 2015), quienes son finalmente los mayores involucrados en el territorio. A partir de lo planteado por Thompson et al. (2011), en cuanto a la comprensión y comunicación de los beneficios de los SE, se debe considerar como parte de los desarrollos metodológicos de nuevas investigaciones: las relaciones sociales, ambientales y económicas que las comunidades construyen con los SE. Esto con el fin de lograr que la toma de decisiones esté cobijada bajo una mirada integral que contribuya a la gestión sostenible de los bosques.

\section{DISCUSIÓN}

Investigaciones recientes han demostrado la reducción en la provisión de SE por cambio de uso del suelo (Hasan et al., 2020; Hanna et al., 2019). 
Así mismo, se ha hecho énfasis en los costos económicos por pérdida directa y por afectación indirecta de servicios de aprovisionamiento y regulación (Sharma et al., 2019). Igualmente, en el contexto del cambio climático, se ha resaltado la importancia de las coberturas naturales en la regulación que mantiene las funciones de agroecosistemas (Organización de las Naciones Unidas para la Alimentación y la Agricultura, 2013). Considerando todo lo anterior, las recomendaciones de manejo planteadas en cuanto a evitar la transformación de las coberturas naturales remanentes son pertinentes.

La inclusión de la percepción de actores locales, con respecto a la importancia de los SE, es una característica relevante de este estudio. Este enfoque participativo se ha manifestado como un proceso necesario para orientar las acciones de manejo en cuencas hidrográficas (Zafra-Calvo et al., 2020); en el caso de los bs-T puede llegar a contribuir en el diseño de intervenciones institucionales apropiadas en el territorio (Castillo et al., 2005). Así mismo, las estrategias de construcción transdisciplinar descentralizadas han sido recomendadas para su incorporación en investigaciones futuras (Jacobs et al., 2020).

En términos generales, los análisis de SE en Colombia se han enfocado en la valoración integral (Rincón-Ruiz et al., 2019a), en la identificación de trade-offs respecto a actividades económicas (Rincón-Ruiz et al., 2019b) y en la modelación en escenarios de cambio climático (Clerici et al., 2019). A pesar de la alta contribución que han proporcionado dichas investigaciones, todavía es necesario profundizar en aspectos como la zonificación, la cual es fundamental en la identificación de relaciones entre los SE y la estrategia espacial de manejo del paisaje (Liu et al., 2019; de Groot et al., 2010). Adicional a lo anterior, la inclusión de un riguroso ejercicio de zonificación puede llegar a ser una herramienta importante en la construcción de procesos de gobernanza, al incluir una visión compartida del territorio con participación local (van Assche et al., 2020).
Esta investigación aporta al conocimiento actual al integrar el enfoque multi-criterio (en lo cartográfico) y multivariado (en lo estadístico), dentro de un análisis de SE. A diferencia de estudios anteriores que solamente han desarrollado su propuesta en bs-T tomando criterios político-administrativos (Andrade et al., 2017) o priorizando un SE (Rincón-Ruiz et al., 2019b). La aproximación aquí realizada permite concentrar las acciones de manejo al zonificar el estado de los SE en el territorio, a una escala semidetallada (1:25 000) (Portillo-Quintero et al., 2015) y relacionar diferentes variables biofísicas de uso del suelo e indicadores del estado de dichos servicios.

Se recalca que la recomendación de manejo, asociada a la integración de los sistemas naturales del área a proyectos de la estrategia REDD+, se sustenta en experiencias previas latinoamericanas en las cuales se haya logrado generar un nicho de desarrollo sostenible en la planeación del paisaje, mediante la conservación de los bosques y la ejecución de actividades agrícolas (Bastos-Lima et al., 2017). Por lo que podría ser una opción interesante para la zona de estudio, siempre y cuando se desarrolle en el marco del respeto por la tenencia de la tierra y por el uso actual del suelo con el fin de evitar la generación de conflictos sociales (Milne et al., 2019). Así mismo, debe considerarse la dificultad del cambio de áreas de cultivo para incrementar los stocks de carbono, teniendo en cuenta que un alto porcentaje de los predios corresponde a pequeños propietarios (Skutsch et al., 2015).

\section{CONCLUSIONES}

El análisis participativo fue esencial para la identificación del alto valor de los SE de regulación hídrica que prestan los ecosistemas naturales y que son clave para la continuidad de las actividades socioeconómicas. En suma, la espacialización de variables biofísicas confirma la importancia de estas áreas en la provisión de SE. 
La declaratoria de los DRMI por parte de las autoridades ambientales es, sin duda, una estrategia que contribuye a la conservación de ecosistemas estratégicos (como el caso del bs-T) y puede ser fortalecida con la incorporación de los elementos aportados en el presente estudio.

La conservación de los ecosistemas naturales debe ser prioritaria. Para darle continuidad a la provisión de los SE de producción agropecuaria y de suministro de agua para acueductos veredales, es necesario cumplir con el planteamiento en mención. Esto teniendo en cuenta que los SE son afectados, de acuerdo con los resultados de la evaluación de los procesos ecosistémicos y la estructura del ecosistema.

\section{AGRADECIMIENTOS}

El presente artículo es producto del Convenio Interadministrativo de Asociación n. ${ }^{\circ} 1594$ de 2016 suscrito por la Corporación Autónoma Regional de Cundinamarca con la Universidad Distrital Francisco José de Caldas. Deseamos agradecer a las alcaldías de los municipios de Nariño, Guataquí, Beltrán, Jerusalén, Pulí y San Juan de Rioseco; a los representantes de las comunidades, líderes y presidentes de las juntas de acción comunal; y a la docente Nelly Rodríguez Eraso de la Universidad Nacional de Colombia por su acompañamiento y asesoría en este trabajo.

\section{CONFLICTOS DE INTERESES}

Los autores declaran no tener conflictos de intereses.

\section{CONTRIBUCIÓN POR AUTOR}

L.M.C.C. y R.L.C. lideraron la investigación. L.M.C.C., R.L.C. y S.D. realizaron muestreos en campo. L.M.C.C., S.D. y R.L.C. hicieron los análisis estadísticos y escribieron el manuscrito. W.G.A. efectuó todos los análisis cartográficos. Todos los autores contribuyeron a la lectura de los borradores.

\section{REFERENCIAS}

Andrade, H. J., Segura, M. A. y Sierra, E. (2017). Percepción local de los servicios ecosistémicos ofertados en fincas agropecuarias de la zona seca del norte del Tolima, Colombia. Revista Luna Azul, 45, 42-58. https://doi.org/10.17151/luaz.2017.45.4

Balvanera, P., Castillo, A. y Martínez-Harms, M.J. (2011). Ecosystem Services in Seasonally Dry Tropical Forests. En R. Dirzo, H. Young, H. Mooney y G. Ceballos (eds.), Seasonally Dry Tropical Forests: Ecology and Conservation (pp. 259-277). Island Press.

Bastos-Lima, M. G., Visseren-Hamakers, I. J., Braña-Varela, J. y Gupta, A. (2017). A reality check on the landscape approach to REDD+: Lessons from Latin America. Forest Policy and Economics, 78, 10-20. https://doi.org/10.1016/j.forpol.2016.12.013

Bubb, P., Soesbergen, A. V., Bisht, N., Singh, G., Joshi, S., Aryal, K., Danks, F. S., Rawat, G. S., Bhuchar, S., Wu, N., Kotru, R. y Yi, S. (2017). Planning Management for Ecosystem Services-An Operations Manual. International Centre for Integrated Mountain Development.

Calvo-Rodríguez, S., Sánchez-Azofeifa, A. G., Durán, S. M. y Espirito-Santo, M. M. (2016). Assessing ecosystem services in Neotropical dry forests: a systematic review. Environmental Conservation, 44, 1, 34-43. https://doi.org/10.1017/S0376892916000400

Castillo, A., Magaña, A., Pujadas, A., Martínez, L. y Godínez, C. (2005). Understanding the Interaction of Rural People with Ecosystems: A Case Study in aTropical Dry Forest of Mexico. Ecosystems, 8, 630-643. https://doi.org/10.1007/s10021-005-0127-1

Clerici, N., Cote-Navarro, F., Escobedo, F. J., Rubiano, K. y Villegas, J. C. (2019). Spatio-temporal and cumulative effects of land use-land cover and climate change on two ecosystem services in the Colombian Andes. 
Science of the Total Environment, 685, 1181-1192.

https://doi.org/10.1016/j.scitotenv.2019.06.275

Corporación Autónoma Regional de Cundinamarca

(CAR) (2006). Caracterización Socioeconómica. En Plan de ordenación y manejo de la cuenca del río Magdalena-vertiente oriental departamento de Cundinamarca (pp. 247-350). CAR.

Corporación Autónoma Regional de Cundinamarca (CAR) (2017). Propuesta de declaratoria de un área protegida en la categoría de distrito de manejo integrado- "Bosque Seco de la Vertiente Oriental, del Río Magdalena en las provincias Centro y Alto Magdalena"- municipios de Nariño, Guataquí, Beltrán, Jerusalén, Pulí, y San Juan de Rio Seco; departamento de Cundinamarca. CAR.

Corporación Autónoma Regional de Cundinamarca (CAR) (17 de julio de 2018). Acuerdo 20 del 17 de julio de 2018. Por medio del cual se declara, delimita y alindera el Distrito Regional de Manejo Integrado "Bosque Seco de la Vertiente Oriental del Rio Magdalena". CAR.

Contraloría de Cundinamarca (2016). Informe anual del estado de los recursos naturales y del ambiente del departamento de Cundinamarca, año 2016-vigencia 2015. Contraloría de Cundinamarca. http://www.contraloriadecundinamarca.gov.co/attachment/002\%20informes/008\%20informe_anual_ del_estado_de_los_recursos_naturales_y_del_ambiente_del_departamento_de_cundinamarca/2016/ consolidado-provincias.html

Crump, M. L. y Scott, N. J. (2001). Relevamientos por encuentros visuales. En R. Heyer, M. D. Donnelly, L. A. McDiarmid, A. Hayek y M. S. Foster (eds.), Medición y monitoreo de la diversidad biológica: métodos estandarizados para anfibios (pp. 80-87). Editorial Universitaria de la Patagonia.

de Groot, R. S., Alkemade, R., Braat, L., Hein, L. y Willemen, L. (2010). Challenges in integrating the concept of ecosystem services and values in landscape planning, management and decision making. Ecological Complexity, 7, 260-272. https://doi.org/10.1016/j.ecocom.2009.10.006

\begin{tabular}{ccccc} 
Environmental & \multicolumn{2}{c}{ Systems } & Research & Insti- \\
tute & (2012). & ArcGIS. & Version & 10.2.
\end{tabular}

Environmental Systems Research Institute. https://www.arcgis.com

Fagerholm, N., Käyhkö, N., Ndumbaro, F. y Khamis, M. (2012). Community stakeholders' knowledge in landscape assessments-Mapping indicators for landscape services. Ecological Indicators, 18, 421-433. https://doi.org/10.1016/j.ecolind.2011.12.004

García, H., Corzo, G., Isaacs, P. y Etter, A. (2014). Capítulo 8: Distribución y estado actual de los remanentes del bioma de bosque seco tropical en Colombia: Insumos para su gestión. En C. Pizano y H. García (eds.), El bosque seco tropical en Colombia (pp. 229-251). Instituto de Investigación de Recursos Biológicos Alexander von Humboldt.

García-Márquez, J. R., Tobias, K., Páez, C. A., Ruiz-Agudelo, C. A., Bejarano, P., Muto, T., y Arjona, F. (2016). Effectiveness of Conservation Areas for Protecting Biodiversity and Ecosystem Services: A Multi-Criteria Approach. International Journal of Biodiversity Science, ECosystem Services \& Management, 13, 1, 1-13. https://doi.org/10.1080/21513732.2016.1200672

Gobernación de Cundinamarca (2016). Base Agrícola 2016. Secretaría de Agricultura, Gobernación de Cundinamarca. http://www.cundinamarca.gov.co/Home/SecretariasEntidades.gc/ Secretariadeagricultura/Secagriculturadespliegue/ asdocumentacion_contenidos/csecreagri_centrodoc_documentos_oficina_asesora_de_planeacion

Gobernación de Cundinamarca (2020). Plan Departamental de Desarrollo 2020-2024, "Cundinamarca, ¡Región que progresa!": Anexo 1 - Diagnóstico por provincias. Gobernación de Cundinamarca.

González-M. R., García, H., Isaacs, P., Cuadros, H., López-Camacho, R., Rodríguez, N., Pérez, K., Mijares, F., Castaño-Naranjo, A., Jurado, R., Idárraga-Piedrahíta, A., Rojas, A., Vergara H. y Pizano, C. (2018). Disentangling the environmental heterogeneity, floristic distinctiveness and current threats of tropical dry forests in Colombia. Environmental Research Letters, 13, 045007. https://doi.org/10.1088/1748-9326/aaad74

Hanna, D. E. L., Raudsepp-Hearne, C. y Bennett, E. M. (2019). Effects of land use, cover, and protection 
on stream and riparian ecosystem services and biodiversity. Conservation Biology, 34, 244-255. https://doi.org/10.1111/cobi.13348

Hasan, S., Shi, W. y Zhu, X. (2020). Impact of land use land cover changes on ecosystem service value - A case study of Guangdong, Hong Kong, and Macao in South China. Plos One, 15, e0231259. https://doi.org/10.1371/journal.pone.0231259

Hoekstra, J. M., Boucher, T. M., Ricketts, T. H. y Cartes, R. (2005). Confronting a biome crisis: global disparities ofhabitatlossand protection. Ecology Letters, 8,23-29. https://doi.org/10.1111/j.1461-0248.2004.00686.x

Holdridge, L. R. (1967). Life zone ecology. Tropical Science Center.

Instituto Geográfico Agustín Codazzi (Igac) (2010). Metodología para los levantamientos de suelos. Igac.

\section{Instituto Geográfico Agustín Codazzi} (Igac) (2017). Datos abiertos Igac. Igac. https://geoportal.igac.gov.co/contenido/datosabiertos-cartografia-y-geografia

Instituto de Hidrología, Meteorología y Estudios Ambientales (Ideam), Instituto Geográfico Agustín Codazzi (Igac) y Corporación Autónoma Regional del río Grande de La Magdalena (Cormagdalena) (2008). Mapa de cobertura de la tierra cuenca Magdalena-Cauca: metodología Corine Land Cover adaptada para Colombia a escala 1:100.000. Ideam, Igac y Cormagdalena.

Instituto de Hidrología, Meteorología y Estudios Ambientales (Ideam) y Ministerio a Ambiente y Desarrollo Sostenible (Mads) (2017). Mapa de ecosistemas continentales, costeros y marinos de Colombia-Escala 1:100000, actualización 2017. Mads, Ideam, IAvH, Sinchi, Invemar, IIAP, PNN e Igac.

Jacobs, S., Zafra-Calvo, N., Gonzalez-Jimenez, D., Guibrunet, L., Benessaiah, K., Berghöfer, A., Chaves-Chaparro, J., Díaz, S., Gomez-Baggethun, E., Lele, S., Martín-López, B., Masterson, V.A., Merçon, J., Moersberger, H., Muraca, B., Norström, A., O'Farrell, P., Ordonez, J. C., Prieur-Richard, A. H. [...] y Balvanera, P. (2020). Use your power for good: plural valuation of nature-the
Oaxaca statement. Global Sustainability, 3, e8. https://doi.org/10.1017/sus.2020.2

Kassambara, A. y Mundt, F. (2018). Package factoextra: Extract and visualize the Results of Multivariate Data Analyses. R Package Version 1.0.7. https://cran.rstudio.com/web/packages/factoextra/ factoextra.pdf

Le, S., Josse, J. y Husson, F. (2008). FactoMineR: An $R$ Package for Multivariate Analysis. Journal of Statistical Software, 25, 1, 1-18. https://doi.org/10.18637/jss.v025.i01

Liu, Y., Li, T., Zhao, W., Wang, S. y Fu, B. (2019). Landscape functional zoning at a county level based on ecosystem services bundle: Methods comparison and management indication. Journal of Environmental Management, 249, 109315. https://doi.org/10.1016/j.jenvman.2019.109315

López-Camacho, R., González-M., R. y Cano, M. (2012). Acacia farnesiana (L.) Willd. (Fabaceae: Leguminosae), una especie exótica con potencial invasivo en los bosques secos de la isla de Providencia (Colombia). Biota Colombiana, 13, 2, 232-246. https://doi.org/10.21068/bc.v13i2.269

López-Camacho, R. y Rojas, J. (eds.) (2019). El bosque seco en el territorio. CAR y Universidad Distrital Francisco José de Caldas.

Maass, J. M., Balvanera, P., Castillo, A., Daily, G. C., Mooney, H. A., Ehrlich, P., Quesada, M., Miranda, A., Jaramillo, V. J., García Oliva, F., Martínez-Yrizar, A., Cotler, H., López-Blanco, J., Pérez-Jiménez, A., Búrqueza, A., Tinoco, C., Ceballos, G., Barraza, L., Ayala, R. y Sarukhán, J. (2005). Ecosystem Services of Tropical Dry Forests: Insights from Longterm Ecological and Social Research on the Pacific Coast of Mexico. Ecology and Society, 10, 1, 17. https://doi.org/10.5751/ES-01219-100117

Mendoza, G. A. y Macoun, P. (1999). Guidelines for Applying Multi-Criteria Analysis to the Assessment of Criteria and Indicators. Center for International Forestry Research. https://doi.org/10.17528/cifor/000769

Millennium Ecosystem Assessment Panel (2005). Ecosystems and Human Well-Being: Synthesis. Island Press. 
Milne, S., Mahanty, S., To, P., Dressler, W., Kanowski, P. y Thavat, M. (2019). Learning from "Actually Existing" REDD+: A Synthesis of EthnographicFindings. ConservationandSociety, 17, 84-95. https://doi.org/10.4103/cs.cs_18_13

Ministerio de Ambiente, Vivienda y Desarrollo Territorial (MAVDT) (1 de julio de 2010). Decreto 2372 de 2010: Por el cual se reglamenta el Decreto Ley 2811 de 1974, la Ley 99 de 1993, la Ley 165 de 1994 y el Decreto Ley 216 de 2003, en relación con el Sistema Nacional de Áreas Protegidas, las categorías de manejo que lo conforman y se dictan otras disposiciones. Diario Oficial N. ${ }^{\circ} 47757$.

Moonlight, P. W., Banda, R. K., Phillips, O. L., Dexter, K. G., Pennington, R. T., Baker, T. R., C. de Lima, H., Fajardo, L., González-M., R., Linares-Palomino, R., Lloyd, J., Nascimento, M., Prado, D., Quintana, C., Riina, R., Rodríguez, G. M., Maria Villela, D., Aquino, A. C. M. M., Arroyo, L., Bezerra, C., Tadeu Brunello, A., [...] y Veenendaal, E. (2020). Expanding tropical forest monitoring into Dry Forests: The DRYFLOR protocol for permanent plots. Plants People Planet, 00, 1-6. https://doi.org/10.1002/ppp3.10112

Organización de las Naciones Unidas para la Alimentación y la Agricultura (FAO) (2013). Climate-Smart: Crop Production System. En Climate-smart agriculture sourcebook (pp. 195-205). FAO.

Paudyal, K., Baral, H. y Keenan, R. J. (2015). Local actions for the common good: Can the application of the ecosystem services concept generate improved societal outcomes from natural resource management? Land Use Policy, 56, 327-332. https://dx.doi.org/10.1016/j.landusepol.2015.11.010

Phelps J., Friess, D. A. y Webb, E. L. (2012). Win-win REDD+ approaches belie carbon-biodiversity trade-offs. Biological Conservation, 154, 53-60. https://doi.org/10.1016/j.biocon.2011.12.031

Pizano C. y García, H. (eds.) (2014). El bosque seco tropical en Colombia. Instituto de Investigación de Recursos Biológicos Alexander von Humboldt.

Portillo-Quintero, C., Sánchez-Azofeifa, A., Calvo-Alvarado, J., Quesada, M. y do Espirito Santo, M. M. (2015). The role of tropical dry forests for biodiversity, carbon and water conservation in the neotropics: lessons learned and opportunities for its sustainable management. Regional Environmental Change, 15, 1039-1049. https://doi.org/10.1007/s10113-014-0689-6

R Development Core Team (2017). R: A language and environment for statistical Computing. The $\mathrm{R}$ Foundation for Statistical Computing. http://www.R-project.org

Ralph, C. J., Geupel, G. R., Pyle, P., Martin, T. E., DeSante, D. F. y Milá, B. (1996). Manual de métodos de campo para el monitoreo de aves terrestres. US Department of Agriculture, Forest Service, Pacific Southwest Research Station.

Reid, F. A. (2009). A Field Guide to the Mammals of Central America and Southeast Mexico. Oxford University Press.

Rincón-Ruiz, A., Arias-Arévalo, P., Núñez Hernández, J. M., Cotler, H., Aguado Caso, M., Meli, P., Tauro, A., Ávila Akerberg, V. D., Avila-Foucat, V. S., Cárdenas, J. P., Castillo Hernández, L. A., Castro, L.G., Cerón Hernández, V. A., Contreras Araque, A., Deschamps-Lomeli, J., Galeana-Pizaña, J. M., Guillén Oñate, K., Hernández Aguilar, J. A., Jimenez, A. D. [...] y Waldron, T. (2019a). Applying integrated valuation of ecosystem services in Latin America: Insights from 21 case studies. Ecosystem Services, 36, 100901. https://doi.org/10.1016/j.ecoser.2019.100901

Rincón-Ruiz, A., Rojas-Padilla, J., Agudelo-Rico, C., Perez-Rincon, M., Vieira-Samper, S. y Rubiano-Páez, J. (2019b). Ecosystem services as an inclusive social metaphor for the analysis and management of environmental conflicts in Colombia. Ecosystem Services, 37, 100924. https://doi.org/10.1016/j.ecoser.2019.100924

Sharma, R., Rimal, B., Baral, H., Nehren, U., Paudyal, K., Sharma, S., Rijal, S., Ranpal, S., Acharya, R., Alenazy, A. y Kandel, P. (2019). Impact of Land Cover Change on Ecosystem Services in a Tropical Forested Landscape. Resources, 8, 18, 1-13. https://doi.org/10.3390/resources8010018

Skutsch, M., Borrego, A., Morales-Barquero, L., Paneque-Gálvez, J., Salinas-Melgoza, M., Ramírez, 
M. I., Perez-Salicrup, D., Benet, D., Monroy, S. y Gao, Y. (2015). Opportunities, constraints and perceptions of rural communities regarding their potential to contribute to forest landscape transitions under REDD+: case studies from Mexico. International Forestry Review, 17, 65-84. https://doi.org/10.1505/146554815814669025

Thompson, I. D., Okabe, K., Tylianakis, J. M., Kumar, P., Brockerhoff, E. G., Schellhorn, N. A., Parrotta, J. A. y Nasi, R. (2011). Forest Biodiversity and the Delivery of Ecosystem Goods and Services: Translating Science into Policy. BioScience, 61, 12, 972-981. https://doi.org/10.1525/bio.2011.61.12.7

Tirira, D. (2007). Mamíferos del Ecuador: guía de campo. Ediciones Murciélago Blanco.

Trejo I. y Dirzo, R. (2000). Deforestation of seasonally dry tropical forest: a national and local analysis in Mexico. Biological Conservation, 94, 2, 133-142. https://doi.org/10.1016/S0006-3207(99)00188-3

van Assche, K., Beunen, R. y Oliveria, E. (2020). Spatial planning and place branding: rethinking relations and synergies. $E u-$ ropean Planning Studies, 28, 7, 1274-1290. https://doi.org/10.1080/09654313.2019.1701289

Vásquez-Valderrama, M. Y., López-Camacho, R., y Baptiste, M. P. (2017). La transformación histórica de las coberturas naturales impulsa el potencial de invasión de plantas en los bosques secos del río Magdalena, Colombia. Biota Colombiana, 18, 2, 132-144. https://doi.org/10.21068/c2017.v18n02a08

Vásquez-Valderrama, M., González-M., R., López-Camacho, R., Baptiste, M. P. y Salgado-Negret, B. (2020). Impact of invasive species on soil hydraulic properties: importance of functional traits. Biological Invasions, 22, 1849-1863. https://doi.org/10.1007/s10530-020-02222-8

Villareal, H., Álvarez, M., Córdoba, S. Escobar, F., Fagua, G., Gast, F., Mendoza, H., Ospina, M. y Umaña, A. M. (2004). Manual de métodos para el desarrollo de inventarios de biodiversidad. Instituto de Investigación de Recursos Biológicos Alexander von Humboldt.

Ward, J. (1963). Hierarchical Grouping to Optimize an Objective Function. Journal of the American Statistical Association, 58, 301, 236-244. https://doi.org/10.2307/2282967

Wold, S., Esbensen, K. y Geladi, P. (1987). Principal Component Analysis. Chenometrics and Intelligent Laboratory Systems, 2, 37-52. https://doi.org/10.1016/0169-7439(87)80084-9

Yirdaw E., Tigabu M. y Monge, A. (2017). Rehabilitation of degraded dryland ecosystems - review. Silva Fennica, 51, 1B, 1673. https://doi.org/10.14214/sf.1673

Zafra-Calvo, N., Balvanera, P., Pascual, U., Merçon, J., Martín-López, B., van Noordwijk, M., Mwampamba, T.H., Lele, S., Ifejika Speranza, C., Arias-Arévalo, P., Cabrol, D., Cáceres, D. M., O'Farrell, P., Subramanian, S. M., Devy, S., Krishnan, S., Carmenta, R., Guibrunet, L., Kraus-Elsin, Y., Moersberger, H., Cariño, J. y Díaz, S. (2020). Plural valuation of nature for equity and sustainability: Insights from the Global South. Global Environmental Change, 63, 102115. https://doi.org/10.1016/j.gloenvcha.2020.102115 


\section{ANEXO SUPLEMENTARIO}

Anexo 1. Valoración de los indicadores asociados al componente fauna. Cobertura según Corine Land Cover

\begin{tabular}{|c|c|c|c|c|c|}
\hline \multirow[b]{2}{*}{ Unidad de análisis } & \multirow[b]{2}{*}{ Cobertura } & \multicolumn{4}{|c|}{ Valoración } \\
\hline & & $\begin{array}{l}\text { Abundancia } \\
\text { de aves } \\
\text { insectívoras }\end{array}$ & Mamíferos & $\begin{array}{c}\text { Abundancia a } \\
\text { nivel del suelo de } \\
\text { descomponedores } \\
\text { como escarabajos } \\
\text { estercoleros }\end{array}$ & $\begin{array}{c}\text { Abundancia } \\
\text { de anfibios y } \\
\text { reptiles }\end{array}$ \\
\hline \multirow{10}{*}{ Ecosistemas naturales } & 3142 & Muy alto & Alto & Muy alto & Muy alto \\
\hline & 31111 & Muy alto & Alto & Muy alto & Muy alto \\
\hline & 3143 & Muy alto & Alto & Muy alto & Muy alto \\
\hline & 3144 & Muy alto & Alto & Muy alto & Muy alto \\
\hline & 32221 & Medio & Medio & Medio & Medio \\
\hline & 32222 & Medio & Medio & Medio & Medio \\
\hline & 3221 & Alto & Muy alto & Alto & Alto \\
\hline & 32122 & Bajo & Bajo & Bajo & Bajo \\
\hline & 3231 & Medio & Medio & Medio & Medio \\
\hline & 3232 & Bajo & Bajo & Bajo & Bajo \\
\hline \multirow{17}{*}{$\begin{array}{l}\text { Agroecosistemas agrí- } \\
\text { colas }\end{array}$} & 211 & Bajo & Bajo & Bajo & Bajo \\
\hline & 2121 & Bajo & Bajo & Medio & Medio \\
\hline & 2122 & Bajo & Bajo & Medio & Medio \\
\hline & 2131 & Bajo & Bajo & Medio & Medio \\
\hline & 2123 & Bajo & Bajo & Medio & Medio \\
\hline & 2132 & Bajo & Bajo & Medio & Medio \\
\hline & 2241 & Alto & Alto & Alto & Alto \\
\hline & 2231 & Muy alto & Muy alto & Muy alto & Muy alto \\
\hline & 2221 & Muy alto & Muy alto & Muy alto & Muy alto \\
\hline & 2215 & Muy alto & Muy alto & Muy alto & Muy alto \\
\hline & 2233 & Medio & Medio & Medio & Medio \\
\hline & 2234 & Medio & Medio & Medio & Medio \\
\hline & 22122 & Medio & Medio & Medio & Medio \\
\hline & 22131 & Medio & Medio & Medio & Medio \\
\hline & 241 & Medio & Medio & Medio & Medio \\
\hline & 245 & Medio & Medio & Medio & Medio \\
\hline & 243 & Medio & Medio & Medio & Medio \\
\hline \multirow{5}{*}{$\begin{array}{l}\text { Agroecosistemas gana- } \\
\text { deros }\end{array}$} & 232 & Muy alto & Muy alto & Muy alto & Muy alto \\
\hline & 233 & Muy alto & Muy alto & Muy alto & Muy alto \\
\hline & 231 & Bajo & Bajo & Bajo & Bajo \\
\hline & 244 & Medio & Medio & Medio & Medio \\
\hline & 242 & Medio & Medio & Medio & Medio \\
\hline
\end{tabular}

Fuente: Ideam, (2008). 
Anexo 2. Valoración de los indicadores asociados al componente suelo

\begin{tabular}{|c|c|c|c|c|c|}
\hline $\begin{array}{l}\text { Perfil } \\
\text { modal }\end{array}$ & $\begin{array}{c}\text { Profundidad } \\
\text { efectiva del suelo }\end{array}$ & $\begin{array}{l}\text { Profundidad de la } \\
\text { capa orgánica del } \\
\text { suelo }\end{array}$ & Unidad de suelos & $\begin{array}{l}\text { Capacidad de uso } \\
\text { por fertilidad }\end{array}$ & $\begin{array}{l}\text { Susceptibilidad } \\
\text { a procesos de } \\
\text { erosión }\end{array}$ \\
\hline 1 & Bajo & Alto & MWSg & Baja & Alta \\
\hline 2 & Alto & Muy alto & MWFf & Media & Alta \\
\hline 3 & Muy bajo & Muy bajo & $M W V f$ & Media & Alta \\
\hline 4 & Medio & Alto & VWQa & Alta & Baja \\
\hline 5 & Medio & Medio & MWFf & Media & Alta \\
\hline 6 & Bajo & Medio & MWCd & Media & Media \\
\hline 7 & Bajo & Muy bajo & MWFf & Media & Alta \\
\hline 8 & Medio & Bajo & MWFf & Media & Alta \\
\hline 9 & Bajo & Muy bajo & MWFf & Media & Alta \\
\hline 10 & Muy alto & Muy alto & MWSg & Baja & Alta \\
\hline 11 & Bajo & Bajo & MWCd & Media & Media \\
\hline 12 & Medio & Alto & MWFf & Media & Alta \\
\hline 13 & Muy bajo & Medio & VWQa & Alta & Baja \\
\hline 14 & Medio & Medio & MWVf & Media & Alta \\
\hline 15 & Medio & Medio & MWJa - MWJc & Alta & Baja \\
\hline 16 & Muy alto & Medio & VWQa & Alta & Baja \\
\hline 17 & Bajo & Muy bajo & MWSg & Baja & Alta \\
\hline 18 & Bajo & Muy bajo & $M W V f$ & Media & Alta \\
\hline 19 & Bajo & Bajo & MWJa - MWJc & Alta & Baja \\
\hline 20 & Muy bajo & Bajo & VWQa & Alta & Baja \\
\hline 21 & Muy bajo & Muy bajo & MWSg & Baja & Alta \\
\hline 22 & Medio & Bajo & $M W V f$ & Media & Alta \\
\hline 23 & Bajo & Bajo & MWCd & Media & Media \\
\hline 24 & Medio & Bajo & MWJa - MWJc & Alta & Baja \\
\hline 25 & Alto & Alto & MWSg & Baja & Alta \\
\hline 26 & Medio & Medio & $M W V f$ & Media & Alta \\
\hline 27 & Muy alto & Alto & MWCd & Media & Media \\
\hline 28 & Alto & Bajo & VWOa & Alta & Baja \\
\hline
\end{tabular}

Fuente: López-Camacho y Rojas (2019). 
Anexo 3. Valoración de los indicadores asociados al componente flora

\begin{tabular}{|c|c|c|c|}
\hline Parcela & $\begin{array}{l}\text { Abundancia de plantas fijadoras de } \\
\text { nitrógeno }\left(\mathrm{n} . .^{\circ} \text { Ind } 0.1 \mathrm{ha}^{-1}\right)\end{array}$ & $\begin{array}{l}\text { Biomasa total por unidad de área } \\
\qquad\left(\mathrm{kg} \mathrm{0.1}^{\left.\mathrm{ha} \mathrm{h}^{-1}\right)}\right.\end{array}$ & $\begin{array}{l}\text { Número de individuos de especies } \\
\text { de flora en categoría de amenaza } \\
\left(\mathrm{n} .^{\circ} \text { Ind } 0.1 \mathrm{ha}^{-1}\right)\end{array}$ \\
\hline 1 & Alto & Medio & Bajo \\
\hline 2 & Muy alto & Muy alto & Muy bajo \\
\hline 3 & Muy bajo & Alto & Muy bajo \\
\hline 4 & Alto & Muy bajo & Medio \\
\hline 5 & Muy bajo & Alto & Muy bajo \\
\hline 6 & Muy bajo & Muy alto & Muy bajo \\
\hline 7 & Muy bajo & Bajo & Muy alto \\
\hline 8 & Bajo & Bajo & Muy bajo \\
\hline 9 & Muy bajo & Medio & Bajo \\
\hline 10 & Muy alto & Bajo & Muy bajo \\
\hline 11 & Muy bajo & Muy bajo & Bajo \\
\hline 12 & Bajo & Alto & Bajo \\
\hline 13 & Muy bajo & Bajo & Muy bajo \\
\hline 14 & Medio & Muy bajo & Muy bajo \\
\hline 15 & Medio & Muy bajo & Muy bajo \\
\hline 16 & Medio & Bajo & Muy bajo \\
\hline 17 & Muy bajo & Alto & Muy bajo \\
\hline 18 & Muy bajo & Alto & Muy bajo \\
\hline
\end{tabular}

Fuente: López-Camacho y Rojas (eds.) (2019). 
Anexo 4. Valoración de los indicadores asociados al componente paisaje. Cobertura según Corine Land Cover

\begin{tabular}{|c|c|c|}
\hline Unidad de análisis & Cobertura & Valoración tipo de cobertura \\
\hline \multirow{10}{*}{ Ecosistemas naturales } & 3142 & Muy alto \\
\hline & 31111 & Muy alto \\
\hline & 3143 & Muy alto \\
\hline & 3144 & Muy alto \\
\hline & 32221 & Alto \\
\hline & 32222 & Alto \\
\hline & 3221 & Muy alto \\
\hline & 32122 & Muy bajo \\
\hline & 3231 & Medio \\
\hline & 3232 & Bajo \\
\hline \multirow{17}{*}{ Agroecosistemas agrícolas } & 211 & Bajo \\
\hline & 2121 & Muy bajo \\
\hline & 2122 & Muy bajo \\
\hline & 2131 & Bajo \\
\hline & 2123 & Bajo \\
\hline & 2132 & Bajo \\
\hline & 2241 & Alto \\
\hline & 2231 & Muy alto \\
\hline & 2221 & Muy alto \\
\hline & 2215 & Muy alto \\
\hline & 2233 & Medio \\
\hline & 2234 & Medio \\
\hline & 22122 & Medio \\
\hline & 22131 & Medio \\
\hline & 241 & Medio \\
\hline & 245 & Medio \\
\hline & 243 & Medio \\
\hline \multirow{5}{*}{ Agroecosistemas ganaderos } & 232 & Muy alto \\
\hline & 233 & Alto \\
\hline & 231 & Bajo \\
\hline & 244 & Medio \\
\hline & 242 & Medio \\
\hline
\end{tabular}

Fuente: Ideam (2008). 
Anexo 5. Características ecológicas de los principales tipos de vegetación en la zona de estudio

\section{Tipo Vegetación}

Vegetación tipo I (Parcela 3) Dominancia de Bursera simaruba $(9.0 \%)$, Trichilia pallida $(6.8 \%)$, Vasconcellea goudotiana (6.3\%), Triplaris americana (5.4\%), Trichilia sp. (5.2\%), Swartzia trianae (4.8\%), Bulnesia carrapo (3.6\%), Simira cordifolia (3.4\%) y Morisonia americana $(3.4 \%)$.

Vegetación tipo II (Parcelas 9, 10, 15 y 17)

Dominancia de Machaerium capote (43.2 \%), Platymiscium hebestachyum (17.5\%), Aspidosperma polyneuron (15.0\%), Astronium graveolens (14.5\%), Trichilia pallida (12.3\%), Cordia alliodora (13.0\%) y Handroanthus ochraceus (12.8\%).
Área basal $=2.468 \mathrm{~m}^{2} 0.1 \mathrm{ha}^{-1}$. Biomasa $=12.510 \mathrm{Mg} 0.1 \mathrm{ha}^{-1}$. Sector con el mayor índice de diversidad $\left(\mathrm{H}^{\prime}=3.401\right.$ y Alpha's Fisher=19.387. Simpson=0.949). Representatividad con respecto al índice de Chao I=68.241. Especies amenazadas: en CR (Oxandra espintana, Gustavia latifolia), EN (Mayna suaveolens, Aspidosperma polyneuron). Especie más abundante: Vasconcellea goudotiana. Otras especies: Trema micrantha, Neea divaricata, Brosimum alicastrum, Amyris pinnata. Otras leguminosas abundantes: Senegalia sp., Inga sp., Platymiscium hebestachyum, Pterocarpus officinalis y Machaerium capote.
Vegetación tipo III (Parcela 5 y 16) Dominancia de Melicoccus bijugatus $(43.0 \%)$, Machaerium goudotii (23.1\%), Aspidosperma polyneuron (14.0\%), Swartzia trianae (12.3\%), Handroanthus ochraceus (8.2 \%), Platymiscium hebestachyum $(7.5 \%)$ y Pithecellobium dulce (6.4\%).
Área basal en rangos de 0.962-1.941 $\mathrm{m}^{2} 0.1 \mathrm{ha}^{-1}$. Biomasa en rangos de 3.797-11.080 Mg 0.1 ha ${ }^{-1}$. Índices de diversidad: $\mathrm{H}^{\prime}=1.555-3.058$ y Alpha's Fisher $=2.872-14.574$, Simpson=0.557-0.913. Bosques dominados por Leguminosas. Representatividad con respecto al índice de Chao I entre 54.376-96.552. Especies amenazadas: en CR (Oxandra espintana), EN (Mayna suaveolens, Aspidosperma polyneuron). Especie más abundante: Aspidosperma polyneuron, Machaerium capote, Eugenia procera y Platymiscium hebestachyum. Otras especies: Trichilia oligofoliolata (Especie endémica), Trichilia carinata (Especie endémica), Casearia corymbosa, Guazuma ulmifolia.
Área basal de 1.275-1.801 m² $0.1 \mathrm{ha}^{-1}$. Biomasa en rangos de 6.286-10.952 Mg 0.1 ha $^{-1}$. Índices de diversidad: $\mathrm{H}^{\prime}=1.856-2.429$ y Alpha's Fisher=8.114, Simpson=0.647-0.858. Bosques de Ilanura de inundación dominados por Melicoccus bijugatus y bosques de terraza aluvial dominados por Machaerium goudotii. Representatividad con respecto al índice de Chao I entre 54.376-96.552. Especies amenazadas: en CR (Oxandra espintana), EN (Aspidosperma polyneuron). Especies endémicas: Pristimera verrucosa. Especie más abundante: Melicoccus bijugatus, Machaerium goudotii. Otras especies: Bursera simaruba y Forsteronia spicata.
Vegetación tipo IV (Parcelas 2, 4, 7, $13,14$ y 18$)$

Dominancia de Zygia cf. longifolia $(49.1 \%)$, Trichilia oligofoliolata (46.4\%), Aspidosperma polyneuron (35.9\%), Astronium graveolens (30.1\%), Machaerium goudotii $(29.7 \%)$, Machaerium capote (21.7\%), Cordia alliodora (19.3\%) y Trichilia pallida $(12.3 \%)$.
Área basal de 1.195-2.283 m² 0.1 ha-1. Biomasa de 4.454-12.507 Mg 0.1 ha-1. Índice de diversidad: $\mathrm{H}^{\prime}=1.298-2.909$ y Alpha's Fisher=3.153-11.423, Simpson=0.533-0.923. Bosques que presentan dominancia de especies de la familia Meliaceae (Trichilia pallida, Trichilia oligofoliolata) y de Leguminosas (Zygia cf. longifolia, Machaerium capote y Machaerium goudotii). Representatividad con respecto al índice de Chao I entre 63.415- 87.552. Especies amenazadas: en CR (Oxandra espintana), EN (Aspidosperma polyneuron, Mayna suaveolens). Especies endémicas: Rinorea marginata, Trichilia oligofoliolata y Trichilia carinata. Especie más abundante: Melicoccus bijugatus, Machaerium goudotii. Otras especies: Rinorea marginata, Bauhinia petiolata, Cynometra sp. Platymiscium hebestachyum, Eugenia procera, Pithecellobium dulce, Triplaris americana, Aspidosperma cuspa, Quadrella odoratissima, Eugenia sp3 y Gustavia verticillata.

Fuente: López-Camacho y Rojas (eds.) (2019). 
Anexo 6. Características ecológicas de los principales grupos de fauna evaluados en la zona de estudio

\begin{tabular}{|c|c|}
\hline Grupo & Diversidad \\
\hline Insectos Coleoptera & $\begin{array}{l}\text { Se colectaron } 163 \text { individuos que corresponden a } 17 \text { morfoespecies de la familia Scarabaeidae } \\
\text { en cuatro localidades de la zona de estudio. Se registraron seis géneros (Canthon, Dichotomius, } \\
\text { Uroxys, Eurystermus, Onthophagus y Phaeneus) de los } 18 \text { registrados en los bosques secos de } \\
\text { Colombia. El género con mayor número de especies registradas fue Canthon, representado por el } \\
52.9 \% \text { de las especies y } 67.5 \% \text { del total de individuos colectados. }\end{array}$ \\
\hline Insectos Lepidoptera & $\begin{array}{l}\text { Se registraron } 52 \text { especies y } 2234 \text { individuos de seis familias (Nymphalidae, Pieridae, Papilionidae, } \\
\text { Lycaenidae, Hesperiidae y Riodinidae) en cuatro localidades de la zona de estudio. La familia más } \\
\text { diversa fue Nymphalidae, con } 27 \text { especies ( } 52 \% \text { ), seguida de la familia Hesperiidae con } 10 \text { espe- } \\
\text { cies. }\end{array}$ \\
\hline Anfibios y Reptiles & $\begin{array}{l}\text { Se registraron } 14 \text { especies que pertenecen a los órdenes Anura y Gymnophiona. Se registraron } 20 \\
\text { especies de reptiles de los órdenes Crocodylia, Testudines y Squamata (nueve especies). }\end{array}$ \\
\hline Aves & $\begin{array}{l}\text { Se registraron } 190 \text { especies de aves distribuidas en } 21 \text { órdenes y } 48 \text { familias. El orden más represen- } \\
\text { tativo fue el de los Passeriformes con } 107 \text { especies y } 19 \text { familias; la familia Tyrannidae fue la más } \\
\text { diversa con } 32 \text { especies, seguida de la familia Thraupidae con } 19 \text { especies e Icteridae con ocho } \\
\text { especies. El segundo orden en representatividad fue el de los Apodiformes con } 13 \text { especies, } 11 \text { de } \\
\text { colibríes y dos especies de vencejos. Se reportaron } 32 \text { especies que se encuentran en la categoría II } \\
\text { de Cites (2017), especies cuyo comercio debe controlarse a fin de evitar una utilización incompati- } \\
\text { ble con su supervivencia. }\end{array}$ \\
\hline Mamíferos & $\begin{array}{l}\text { Se registraron un total de } 28 \text { especies de mamíferos. Las especies reportadas están distribuidas en } \\
\text { ocho órdenes y } 16 \text { familias. Se encontró una alta diversidad en el grupo del orden Chiroptera con } \\
14 \text { especies, entre las que se encuentran especies frugívoras, insectívoras, hematófagas y nectarívo- } \\
\text { ras. }\end{array}$ \\
\hline
\end{tabular}

Fuente: López-Camacho y Rojas (eds.) (2019). 
Anexo 7. Guía de entrevista semiestructurada.

\section{Guía de preguntas}

\begin{tabular}{|c|c|c|}
\hline 1 & Cultivos & $\begin{array}{l}\text { ¿Usted o su familia cultivan algún producto? ¿Cuáles productos? ¿En qué } \\
\text { áreas? ¿Aproximación a la rentabilidad? }\end{array}$ \\
\hline 2 & Ganado & $\begin{array}{l}\text { ¿Usted o su familia tienen ganado? ¿Cuántas cabezas? ¿De qué tipo? ¿Qué } \\
\text { áreas usan para pastoreo? ¿Aproximación a la rentabilidad? }\end{array}$ \\
\hline 3 & Frutos silvestres & $\begin{array}{l}\text { ¿Usted o su familia colecta frutos o vegetales silvestres? ¿Cuáles? ¿En qué } \\
\text { cantidad? ¿Con qué frecuencia? ¿En qué áreas? }\end{array}$ \\
\hline 4 & Cacería & $\begin{array}{l}\text { ¿Usted o su familia cazan? ¿Qué especies? ¿Qué cantidad? ¿Con qué } \\
\text { frecuencia? ¿En qué áreas? ¿Aproximación a la rentabilidad? }\end{array}$ \\
\hline 5 & Pesca & $\begin{array}{l}\text { ¿Usted o su familia pescan? ¿Qué especies? ¿Qué cantidad? ¿Con qué } \\
\text { frecuencia? ¿En qué áreas? ¿Aproximación a la rentabilidad? }\end{array}$ \\
\hline 6 & Apicultura & $\begin{array}{l}\text { ¿Usted o su familia practican la apicultura? ¿Apiarios o miel silvestre? ¿En } \\
\text { qué cantidad (número de colmenas)? ¿En qué áreas? ¿Aproximación a la } \\
\text { rentabilidad? }\end{array}$ \\
\hline 7 & Leña & $\begin{array}{l}\text { ¿Usa leña en la casa? ¿Qué cantidad? ¿Con qué frecuencia corta la misma } \\
\text { cantidad? ¿En qué áreas corta la leña? ¿Usa o fabrica carbón vegetal? }\end{array}$ \\
\hline 8 & $\begin{array}{l}\text { Flores o materiales } \\
\text { decorativos }\end{array}$ & $\begin{array}{l}\text { ¿Usted usa flores u otros materiales de la naturaleza para propósitos } \\
\text { decorativos? ¿En qué cantidad? ¿Con qué frecuencia? }\end{array}$ \\
\hline 9 & Plantas medicinales & $\begin{array}{l}\text { ¿Colecta plantas medicinales? ¿Cuáles especies? ¿Para qué enfermedad? ¿En } \\
\text { cuales áreas las colecta? }\end{array}$ \\
\hline 10 & Especias & $\begin{array}{l}\text { ¿Colecta plantas especias (condimentos)? ¿Cuáles especies? ¿En qué cantidad? } \\
\text { ¿En cuales áreas las colecta? }\end{array}$ \\
\hline 11 & Plantación de árboles & $\begin{array}{l}\text { ¿Está involucrado en la plantación de árboles? ¿En qué áreas? ¿En qué } \\
\text { cantidades? }\end{array}$ \\
\hline 12 & Madera & $\begin{array}{l}\text { ¿Colecta o corta materiales para construcción, canoas, postes o muebles del } \\
\text { bosque natural? ¿Cuáles especies? ¿Qué cantidades? ¿Con qué frecuencia? } \\
\text { ¿En cuáles áreas? }\end{array}$ \\
\hline 13 & Artesanías & $\begin{array}{l}\text { ¿Colecta o corta materiales para artesanías del bosque natural? ¿Cuáles } \\
\text { especies? ¿Qué cantidades? ¿Con qué frecuencia? ¿En cuales áreas? }\end{array}$ \\
\hline 14 & $\begin{array}{l}\text { Colorantes, resinas, } \\
\text { aceites }\end{array}$ & $\begin{array}{l}\text { ¿Colecta o corta otros productos del bosque natural (tintes o colorantes, } \\
\text { resinas, aceites)? ¿Cuáles especies? ¿Qué cantidades? ¿Con qué frecuencia? } \\
\text { ¿En cuales áreas? }\end{array}$ \\
\hline 15 & Origen del agua & ¿De dónde obtiene el agua para la casa y para la finca? \\
\hline 16 & Lugares sagrados & $\begin{array}{l}\text { ¿Hay lugares religiosos o sagrados para usted en la naturaleza? ¿Cuáles y } \\
\text { dónde? ¿Le da algún sentimiento religioso o espiritual o valor específico a ese } \\
\text { sitio específico? }\end{array}$ \\
\hline 17 & Sitios atractivos & ¿Cuáles sitios son los más hermosos y atractivos aquí? \\
\hline 18 & Valores culturales & $\begin{array}{l}\text { ¿Hay áreas específicas de culturas, tradiciones o sabidurías locales que usted } \\
\text { aprecie y piense que son importantes? }\end{array}$ \\
\hline 19 & $\begin{array}{c}\text { Sitios para la } \\
\text { conservación del agua }\end{array}$ & ¿Cuáles son las áreas de mayor importancia para la conservación del agua? \\
\hline 20 & $\begin{array}{l}\text { Sitios con procesos de } \\
\text { erosión }\end{array}$ & $\begin{array}{l}\text { ¿Cuáles áreas presentan procesos de erosión o han presentado eventos de } \\
\text { deslizamientos o remoción en masa? }\end{array}$ \\
\hline
\end{tabular}




\begin{tabular}{|c|c|l|}
\hline 21 & Sitios productivos & $\begin{array}{l}\text { ¿Cuáles áreas eran productivas para algún tipo de cultivo y actualmente no lo } \\
\text { son? }\end{array}$ \\
\hline 22 & $\begin{array}{c}\text { Sitios bocatomas } \\
\text { acueductos veredales }\end{array}$ & $\begin{array}{l}\text { ¿Dónde están las bocatomas de los acueductos veredales y en qué estado de } \\
\text { conservación se encuentra el área de abastecimiento y recarga de dicha fuente } \\
\text { hídrica? }\end{array}$ \\
\hline 23 & $\begin{array}{c}\text { Sitios para avistamiento } \\
\text { de aves }\end{array}$ & $\begin{array}{l}\text { ¿Cuáles son las zonas de avistamiento de especies como aves o alguna especie } \\
\text { de interés científico? }\end{array}$ \\
\hline 24 & Forrajeras & ¿Conoce o usa especies forrajeras? \\
\hline
\end{tabular}

Actores clave entrevistados

\section{San Juan de Río Seco:}

Vereda Capote:

Integrante de la JAC (7)

Vereda Santa Rosa

Presidente de la JAC (6)

\section{Beltrán:}

Finca La Morada:

Campesino (8)

Presidente de la JAC vereda El Tabor (4)

Presidente de la JAC vereda Chacara (5)

Funcionaria de Alcaldía Municipal (11)

\section{Jerusalén:}

Vereda El Hatillo,

Comerciante (1)

Vereda El Hatillo,

Campesino (2)

Vereda El Bebedero,

Presidente de la JAC (3)

\section{Guataquí:}

Cabecera municipal:

Artesano (9)

Cabecera municipal:

Campesino (10) 
Anexo 8. Resultados de las entrevistas semiestructuradas

\begin{tabular}{|c|c|c|c|c|c|c|c|c|c|c|c|c|c|c|c|}
\hline \multirow{2}{*}{ SE } & \multirow{2}{*}{ Pregunta } & Entrevista & \multirow{2}{*}{1} & \multirow{2}{*}{2} & \multirow{2}{*}{3} & \multirow{2}{*}{4} & \multirow{2}{*}{5} & \multirow{2}{*}{6} & \multirow{2}{*}{7} & \multirow{2}{*}{8} & \multirow{2}{*}{9} & \multirow{2}{*}{10} & \multirow{2}{*}{11} & \multirow{2}{*}{$\begin{array}{l}\text { Total } \\
\text { general }\end{array}$} & \multirow{2}{*}{$\%$} \\
\hline & & Tema & & & & & & & & & & & & & \\
\hline Aprovisionamiento & 1 & Cultivos & 1 & 1 & 1 & 1 & 1 & 1 & 1 & 1 & 1 & 1 & 1 & 11 & 100 \\
\hline Aprovisionamiento & 2 & Ganado & 1 & 1 & 1 & 1 & 1 & 1 & 1 & 1 & 1 & 1 & & 10 & 91 \\
\hline Aprovisionamiento & 12 & Madera & 1 & 1 & 1 & 1 & 1 & 1 & 1 & 1 & 1 & 1 & & 10 & 91 \\
\hline Regulación & 21 & $\begin{array}{l}\text { Sitios para la conservación del } \\
\text { agua }\end{array}$ & 1 & 1 & 1 & 1 & 1 & 1 & 1 & 1 & 1 & & 1 & 10 & 91 \\
\hline Culturales & 17 & Sitios atractivos & 1 & 1 & 1 & 1 & 1 & 1 & & & 1 & 1 & 1 & 9 & 82 \\
\hline Aprovisionamiento & 3 & Frutos silvestres & 1 & 1 & & 1 & 1 & 1 & & 1 & 1 & 1 & & 8 & 73 \\
\hline Aprovisionamiento & 4 & Cacería & 1 & 1 & & 1 & 1 & 1 & & & 1 & 1 & & 7 & 64 \\
\hline Aprovisionamiento & 5 & Pesca & 1 & 1 & & 1 & 1 & 1 & & & 1 & & 1 & 7 & 64 \\
\hline Aprovisionamiento & 7 & Leña & 1 & 1 & 1 & 1 & & 1 & & 1 & 1 & & & 7 & 64 \\
\hline Aprovisionamiento & 9 & Plantas medicinales & 1 & 1 & & 1 & & 1 & & & 1 & 1 & & 6 & 55 \\
\hline Aprovisionamiento & 13 & Artesanías & 1 & 1 & & 1 & & 1 & & & 1 & 1 & & 6 & 55 \\
\hline Aprovisionamiento & 8 & Flores o materiales decorativos & 1 & 1 & & 1 & & 1 & & & 1 & & & 5 & 45 \\
\hline Aprovisionamiento & 14 & Colorantes, resinas, aceites & 1 & 1 & & 1 & 1 & 1 & & & & & & 5 & 45 \\
\hline Culturales & 16 & Lugares sagrados & 1 & 1 & & 1 & & 1 & & & & & 1 & 5 & 45 \\
\hline Aprovisionamiento & 6 & Apicultura & 1 & 1 & & 1 & & 1 & & & & & & 4 & 36 \\
\hline Aprovisionamiento & 10 & Especias & 1 & 1 & & 1 & & 1 & & & & & & 4 & 36 \\
\hline Aprovisionamiento & 11 & Plantación de árboles & 1 & 1 & & 1 & & 1 & & & & & & 4 & 36 \\
\hline Aprovisionamiento & 15 & Origen del agua & 1 & 1 & & 1 & & 1 & & & & & & 4 & 36 \\
\hline Regulación & 20 & Sitios con procesos de erosión & & & & 1 & & 1 & & & & & & 2 & 18 \\
\hline Culturales & 18 & Valores culturales & & & & & & & & & & & 1 & 1 & 9 \\
\hline Regulación & 21 & Sitios productivos & & & & & & 1 & & & & & & 1 & 9 \\
\hline Aprovisionamiento & 22 & $\begin{array}{l}\text { Sitios bocatomas acueductos } \\
\text { veredales }\end{array}$ & & & & & & 1 & & & & & & 1 & 9 \\
\hline Culturales & 23 & Sitios para avistamiento de aves & & & & 1 & & & & & & & & 1 & 9 \\
\hline Aprovisionamiento & 24 & Forrajeras & & & & & & & & & & 1 & & 1 & 9 \\
\hline \multicolumn{3}{|c|}{ Total general } & 18 & 18 & 6 & 20 & 9 & 21 & 4 & 6 & 12 & 9 & 6 & 129 & \\
\hline
\end{tabular}


Anexo 9. 84 puntos de observación de impulsores de cambio.

\begin{tabular}{|c|c|c|c|c|c|c|c|}
\hline $\begin{array}{c}\text { Código } \\
\text { de } \\
\text { punto }\end{array}$ & Latitud & Longitud & \begin{tabular}{|c|}
$\begin{array}{c}\text { Cobertura } \\
\text { de bosque } \\
\text { maduro }\end{array}$ \\
\end{tabular} & $\begin{array}{c}\text { Cobertura } \\
\text { de bosque } \\
\text { secundario }\end{array}$ & \begin{tabular}{|c|}
$\begin{array}{c}\text { Cobertura } \\
\text { de } \\
\text { rastrojos }\end{array}$ \\
\end{tabular} & $\begin{array}{l}\text { Presiones } \\
\text { dentro del } \\
\text { fragmento }\end{array}$ & $\begin{array}{l}\text { Presiones en la matriz del } \\
\text { fragmento }\end{array}$ \\
\hline 1 & $4^{\circ} 17^{\prime} 25.5^{\prime \prime}$ & $74^{\circ} 50^{\prime} 22.9^{\prime \prime}$ & 0 & 80 & 20 & $\begin{array}{l}\text { Ganadería, } \\
\text { quemas. }\end{array}$ & $\begin{array}{l}\text { Cultivos agropecuarios (algo- } \\
\text { dón y sorgo). }\end{array}$ \\
\hline 2 & $4^{\circ} 17^{\prime} 32.7^{\prime \prime}$ & $74^{\circ} 50^{\prime} 38.6^{\prime \prime}$ & 0 & 0 & 100 & & Cultivos (maíz), ganadería. \\
\hline 3 & $4^{\circ} 17^{\prime} 26.7^{\prime \prime}$ & $74^{\circ} 50^{\prime} 35^{\prime \prime}$ & 0 & 100 & 0 & & $\begin{array}{l}\text { Tala selectiva (caracolí), } \\
\text { cultivos agropecuarios (sorgo), } \\
\text { ganadería. }\end{array}$ \\
\hline 4 & $4^{\circ} 19^{\prime} 35^{\prime \prime}$ & $74^{\circ} 51^{\prime} 39.7^{\prime \prime}$ & 0 & 20 & 80 & Erosión. & $\begin{array}{l}\text { Actividades mineras o can- } \\
\text { teras, cultivos agropecuarios } \\
\text { (sorgo), ganadería. }\end{array}$ \\
\hline 5 & $4^{\circ} 25^{\prime} 38.2^{\prime \prime}$ & $74^{\circ} 49^{\prime} 50.1^{\prime \prime}$ & 0 & 0 & 100 & $\begin{array}{l}\text { Infraestructura } \\
\text { humana (basu- } \\
\text { rero, urbaniza- } \\
\text { ción). } \\
\end{array}$ & $\begin{array}{l}\text { Infraestructura humana (urba- } \\
\text { nización). }\end{array}$ \\
\hline 6 & $4^{\circ} 32^{\prime} 0.14^{\prime \prime}$ & $74^{\circ} 46^{\prime} 40.2^{\prime \prime}$ & 0 & 0 & 100 & $\begin{array}{l}\text { Tala selectiva } \\
\text { (carbón), gana- } \\
\text { dería. }\end{array}$ & $\begin{array}{l}\text { Actividades mineras o canteras } \\
\text { (extracción de arena), cultivos } \\
\text { agropecuarios (maíz), gana- } \\
\text { dería. }\end{array}$ \\
\hline 7 & $5^{\circ} 10^{\prime} 6.49^{\prime \prime}$ & $74^{\circ} 47^{\prime} 8.04^{\prime \prime}$ & 65 & 35 & 0 & $\begin{array}{l}\text { Infraestructura } \\
\text { humana (bebe- } \\
\text { deros, tubería } \\
\text { de agua). } \\
\end{array}$ & $\begin{array}{l}\text { Ganadería, infraestructura } \\
\text { humana (bebederos). }\end{array}$ \\
\hline 8 & $5^{\circ} 09^{\prime} 9.83^{\prime \prime}$ & $74^{\circ} 48^{\prime} 6.20^{\prime \prime}$ & 100 & 0 & 0 & & Ganadería. \\
\hline 9 & $4^{\circ} 47^{\prime} 53.6^{\prime \prime}$ & $74^{\circ} 46^{\prime} 18.1^{\prime \prime}$ & 0 & 40 & 60 & Ganadería. & $\begin{array}{l}\text { Actividades mineras o can- } \\
\text { teras, cultivos agropecuarios } \\
\text { (arroz). }\end{array}$ \\
\hline 10 & $4^{\circ} 45^{\prime} 54^{\prime \prime}$ & $74^{\circ} 47^{\prime} 49.1^{\prime \prime}$ & 0 & 100 & 0 & $\begin{array}{l}\text { Claros (caída de } \\
\text { árboles), cami- } \\
\text { nos veredales. }\end{array}$ & Cultivos agropecuarios (arroz). \\
\hline 11 & $4^{\circ} 43^{\prime} 48.5^{\prime \prime}$ & $74^{\circ} 49^{\prime} 14.4^{\prime \prime}$ & 0 & 0 & 100 & & \\
\hline 12 & $4^{\circ} 44^{\prime} 48.8^{\prime \prime}$ & $74^{\circ} 49^{\prime} 25.2^{\prime \prime}$ & 0 & 0 & 100 & $\begin{array}{l}\text { Infraestructura } \\
\text { humana (cana- } \\
\text { les para arroz), } \\
\text { tala selectiva, } \\
\text { ganadería. } \\
\end{array}$ & $\begin{array}{l}\text { Cultivos agropecuarios (arroz), } \\
\text { Infraestructura humana (carre- } \\
\text { tera). }\end{array}$ \\
\hline 13 & $4^{\circ} 40^{\prime} 16.3^{\prime \prime}$ & $74^{\circ} 57^{\prime} 12.4^{\prime \prime}$ & 0 & 15 & 0 & $\begin{array}{l}\text { Ganadería } \\
\text { (ramoneo). }\end{array}$ & Ganadería. \\
\hline 14 & $4^{\circ} 33^{\prime} 29.8^{\prime \prime}$ & $74^{\circ} 55^{\prime} 48^{\prime \prime}$ & 0 & 90 & 10 & $\begin{array}{l}\begin{array}{l}\text { Ganadería } \\
\text { (ramoneo). }\end{array} \\
\end{array}$ & $\begin{array}{l}\text { Ganadería, infraestructura hu- } \\
\text { mana (carretera a piedras). } \\
\end{array}$ \\
\hline 15 & $4^{\circ} 31^{\prime} 34.5^{\prime \prime}$ & $74^{\circ} 53^{\prime} 45.7^{\prime \prime}$ & 0 & 100 & 0 & $\begin{array}{l}\text { Ganadería } \\
\text { (ramoneo). }\end{array}$ & $\begin{array}{l}\text { Cultivos agropecuarios (pláta- } \\
\text { no y yuca), ecoturismo. }\end{array}$ \\
\hline 16 & $4^{\circ} 53^{\prime} 57.5^{\prime \prime}$ & $74^{\circ} 46^{\prime} 20.3^{\prime \prime}$ & 0 & 100 & 0 & $\begin{array}{l}\text { Ganadería y } \\
\text { tala selectiva. }\end{array}$ & $\begin{array}{l}\text { Ganadería, agricultura, que- } \\
\text { mas, infraestructura humana } \\
\text { (vías). }\end{array}$ \\
\hline
\end{tabular}


Análisis participativo de servicios ecosistémicos en un área protegida del bosque seco tropical (bs-T), Colombia

Cárdenas-Camacho, L. M., Díaz, S., Gómez-Anaya, W., Rojas-Rojas J. E. y lópez-Camacho, R.

\begin{tabular}{|c|c|c|c|c|c|c|c|}
\hline 17 & $4^{\circ} 53^{\prime} 23.9^{\prime \prime}$ & $74^{\circ} 42^{\prime} 41.0^{\prime \prime}$ & 20 & 60 & 20 & $\begin{array}{l}\text { Infraestructura } \\
\text { humana (sende- } \\
\text { ros, basura). }\end{array}$ & $\begin{array}{l}\text { Infraestructura humana (vías), } \\
\text { tala selectiva. }\end{array}$ \\
\hline 18 & $4^{\circ} 52^{\prime} 39.1^{\prime \prime}$ & $74^{\circ} 44^{\prime} 00.9^{\prime \prime}$ & 0 & 0 & 100 & $\begin{array}{l}\text { Ganadería e } \\
\text { infraestructura } \\
\text { humana. }\end{array}$ & $\begin{array}{l}\text { Agricultura (arroz y algodón), } \\
\text { infraestructura humana. }\end{array}$ \\
\hline 19 & $4^{\circ} 39^{\prime} 38.6^{\prime \prime}$ & $74^{\circ} 46^{\prime} 42.8^{\prime \prime}$ & 0 & 100 & 0 & $\begin{array}{l}\text { Infraestructura } \\
\text { humana (vías) y } \\
\text { ganadería. }\end{array}$ & $\begin{array}{l}\text { Infraestructura humana (vías) y } \\
\text { ganadería. }\end{array}$ \\
\hline 20 & $4^{\circ} 41^{\prime} 37.4^{\prime \prime}$ & $74^{\circ} 42^{\prime} 15.5^{\prime \prime}$ & 0 & 10 & 0 & $\begin{array}{l}\text { Agricultura e } \\
\text { Infraestructura } \\
\text { humana. }\end{array}$ & $\begin{array}{l}\text { Agricultura (maíz, plátano) } \\
\text { ganadería e Infraestructura } \\
\text { humana (vías). }\end{array}$ \\
\hline 21 & $4^{\circ} 40^{\prime} 10.2^{\prime \prime}$ & $74^{\circ} 43^{\prime} 20.7^{\prime \prime}$ & 0 & 10 & 0 & $\begin{array}{l}\text { Ganadería y } \\
\text { agricultura. }\end{array}$ & $\begin{array}{l}\text { Infraestructura humana (vías) y } \\
\text { agricultura (plátano, maíz). }\end{array}$ \\
\hline 22 & $4^{\circ} 37^{\prime} 38.0^{\prime \prime}$ & $74^{\circ} 44^{\prime} 13.6^{\prime \prime}$ & 0 & 0 & 100 & $\begin{array}{l}\text { Ganadería, } \\
\text { explotación de } \\
\text { hidrocarburos y } \\
\text { quemas. }\end{array}$ & $\begin{array}{l}\text { Infraestructura humana (vías), } \\
\text { ganadería, explotación de } \\
\text { hidrocarburos y quemas. }\end{array}$ \\
\hline 23 & $4^{\circ} 37^{\prime} 57.3^{\prime \prime}$ & $74^{\circ} 45^{\prime} 30.6^{\prime \prime}$ & 0 & 10 & 90 & $\begin{array}{l}\text { Tala intensiva } \\
\text { e infraestruc- } \\
\text { tura humana } \\
\text { (basura). }\end{array}$ & $\begin{array}{l}\text { Infraestructura humana (vías), } \\
\text { explotación de hidrocarburos } \\
\text { y quemas. }\end{array}$ \\
\hline 24 & $4^{\circ} 33^{\prime} 25.0^{\prime \prime}$ & $74^{\circ} 48^{\prime} 04.7^{\prime \prime}$ & 0 & 100 & 0 & $\begin{array}{l}\text { Tala intensiva } \\
\text { (quemas), In- } \\
\text { fraestructura hu- } \\
\text { mana (basura) y } \\
\text { ganadería. }\end{array}$ & $\begin{array}{l}\text { Infraestructura humana (vías), } \\
\text { ganadería y extracción de } \\
\text { canteras. }\end{array}$ \\
\hline 25 & $4^{\circ} 10^{\prime} 16.1^{\prime \prime}$ & $74^{\circ} 41^{\prime} 40.4^{\prime \prime}$ & 0 & 0 & 100 & $\begin{array}{l}\text { Infraestruc- } \\
\text { tura humana } \\
\text { (conjuntos } \\
\text { recreacionales), } \\
\text { bañadero muni- } \\
\text { cipal. }\end{array}$ & $\begin{array}{l}\text { Infraestructura humana (fincas } \\
\text { recreacionales), ganadería. }\end{array}$ \\
\hline 26 & $4^{\circ} 09^{\prime} 36.8^{\prime \prime}$ & $74^{\circ} 42^{\prime} 17.5^{\prime \prime}$ & 0 & 0 & 100 & $\begin{array}{l}\text { Ganadería } \\
\text { (ramoneo). }\end{array}$ & $\begin{array}{l}\text { Ganadería e infraestructura } \\
\text { humana (conjuntos recreacio- } \\
\text { nales). }\end{array}$ \\
\hline 27 & $4^{\circ} 08^{\prime} 12.8^{\prime \prime}$ & $74^{\circ} 41^{\prime} 23.1^{\prime \prime}$ & 0 & 0 & 100 & Quemas. & Ganadería y canteras. \\
\hline 28 & $4^{\circ} 08^{\prime} 01.8^{\prime \prime}$ & $74^{\circ} 41^{\prime} 03.7^{\prime \prime}$ & 0 & 80 & 20 & $\begin{array}{l}\text { Infraestructura } \\
\text { humana (tubos } \\
\text { de acueducto, } \\
\text { basura). } \\
\end{array}$ & Ganadería. \\
\hline 29 & $4^{\circ} 02^{\prime} 51.3^{\prime \prime}$ & $74^{\circ} 41^{\prime} 58.4^{\prime \prime}$ & 0 & 0 & 100 & $\begin{array}{l}\text { Sin uso. Des- } \\
\text { canso. }\end{array}$ & Ganadería y cultivos de maíz. \\
\hline 30 & $4^{\circ} 03^{\prime} 44.9^{\prime \prime}$ & $74^{\circ} 41^{\prime} 20.9^{\prime \prime}$ & 0 & 0 & 100 & $\begin{array}{l}\text { Agricultura (plá- } \\
\text { tano y cacao). }\end{array}$ & $\begin{array}{l}\text { Infraestructura humana (zonas } \\
\text { suburbanas). }\end{array}$ \\
\hline 31 & $4^{\circ} 31^{\prime} 34.5^{\prime \prime}$ & $74^{\circ} 53^{\prime} 45.7^{\prime \prime}$ & 0 & 0 & 100 & $\begin{array}{l}\text { Ganadería } \\
\text { (ramoneo) e } \\
\text { infraestructura } \\
\text { humana (tubería } \\
\text { para extracción } \\
\text { de agua). }\end{array}$ & $\begin{array}{l}\text { Ganadería e infraestructura } \\
\text { humana (carretera). }\end{array}$ \\
\hline
\end{tabular}




\begin{tabular}{|c|c|c|c|c|c|c|c|}
\hline 32 & $4^{\circ} 14^{\prime} 57.9^{\prime \prime}$ & $74^{\circ} 46^{\prime} 00.3^{\prime \prime}$ & 0 & 0 & 100 & $\begin{array}{l}\text { Ganadería } \\
\text { (bebederos) } \\
\text { y presencia } \\
\text { de basura por } \\
\text { arrastre agua. }\end{array}$ & $\begin{array}{l}\text { Ganadería e infraestructura } \\
\text { humana (carretera). }\end{array}$ \\
\hline 33 & $2^{\circ} 44^{\prime} 19.9^{\prime \prime}$ & $75^{\circ} 18^{\prime} 47.4^{\prime \prime}$ & 0 & 0 & 100 & $\begin{array}{l}\text { Infraestruc- } \\
\text { tura humana } \\
\text { (extracción de } \\
\text { arena, hornos } \\
\text { para secado de } \\
\text { tabaco). }\end{array}$ & Ganadería, pequeños cultivos. \\
\hline 34 & $2^{\circ} 39^{\prime} 39.1^{\prime \prime}$ & $75^{\circ} 18^{\prime} 31.7^{\prime \prime}$ & 0 & 0 & 100 & $\begin{array}{l}\text { Ganadería } \\
\text { (bebederos) y } \\
\text { agricultura (pe- } \\
\text { queñas parcelas } \\
\text { de plátano). }\end{array}$ & $\begin{array}{l}\text { Ganadería e infraestructura } \\
\text { humana (invasiones). }\end{array}$ \\
\hline 35 & $2^{\circ} 35^{\prime} 15.2^{\prime \prime}$ & $75^{\circ} 21^{\prime} 41.9^{\prime \prime}$ & 0 & 0 & 100 & Ganadería. & Ganadería. \\
\hline 36 & $2^{\circ} 35^{\prime} 01.1^{\prime \prime}$ & $75^{\circ} 26^{\prime} 53.5^{\prime \prime}$ & 0 & 20 & 0 & $\begin{array}{l}\text { Infraestructura } \\
\text { humana (extrac- } \\
\text { ción de material } \\
\text { de río y presen- } \\
\text { cia de barrios } \\
\text { periféricos). }\end{array}$ & $\begin{array}{l}\text { Infraestructura humana (cante- } \\
\text { ras y cabecera municipal). }\end{array}$ \\
\hline 37 & $2^{\circ} 41^{\prime} 45.8^{\prime \prime}$ & $75^{\circ} 17^{\prime} 32.6^{\prime \prime}$ & 0 & 0 & 100 & Ganadería. & $\begin{array}{l}\text { Ganadería y agricultura (huer- } \\
\text { tos caseros). }\end{array}$ \\
\hline 38 & $2^{\circ} 44^{\prime} 30.6^{\prime \prime}$ & $75^{\circ} 15^{\prime} 27.4^{\prime \prime}$ & 0 & 0 & 100 & $\begin{array}{l}\text { Ganadería y } \\
\text { áreas en des- } \\
\text { canso. }\end{array}$ & Ganadería y quemas. \\
\hline 39 & $2^{\circ} 47^{\prime} 47.2^{\prime \prime}$ & $75^{\circ} 14^{\prime} 30.5^{\prime \prime}$ & 0 & 100 & 0 & $\begin{array}{l}\text { Infraestructura } \\
\text { humana (tubería } \\
\text { para extrac- } \\
\text { ción de agua } \\
\text { y presencia de } \\
\text { basura). }\end{array}$ & $\begin{array}{l}\text { Infraestructura humana (fincas } \\
\text { recreacionales), ganadería. }\end{array}$ \\
\hline 40 & $2^{\circ} 45^{\prime} 42.0^{\prime \prime}$ & $75^{\circ} 14^{\prime} 08.3^{\prime \prime}$ & 0 & 0 & 100 & $\begin{array}{l}\text { Ganadería e } \\
\text { Infraestructura } \\
\text { humana (tubos } \\
\text { para extracción } \\
\text { de agua). }\end{array}$ & Ganadería. \\
\hline 41 & $2^{\circ} 52^{\prime} 04.4^{\prime \prime}$ & $75^{\circ} 13^{\prime} 15.6^{\prime \prime}$ & 0 & 100 & 0 & $\begin{array}{l}\text { Ganadería y } \\
\text { caminos indí- } \\
\text { genas. }\end{array}$ & $\begin{array}{l}\text { Ganadería e infraestructura } \\
\text { humana (barrios suburbanos, } \\
\text { basura). }\end{array}$ \\
\hline 42 & $2^{\circ} 53^{\prime} 11.9^{\prime \prime}$ & $75^{\circ} 23^{\prime} 05.8^{\prime \prime}$ & 60 & 40 & 0 & $\begin{array}{l}\text { Infraestruc- } \\
\text { tura humana } \\
\text { (mangueras para } \\
\text { extracción de } \\
\text { agua). }\end{array}$ & $\begin{array}{l}\text { Ganadería, quemas e infraes- } \\
\text { tructura (molino de caliza) y } \\
\text { áreas enrastrojadas. }\end{array}$ \\
\hline 43 & $2^{\circ} 53^{\prime} 12.7^{\prime \prime}$ & $75^{\circ} 23^{\prime} 02.5^{\prime \prime}$ & 0 & 0 & 100 & Quemas. & Ganadería. \\
\hline 44 & $2^{\circ} 52^{\prime} 57.1^{\prime \prime}$ & $75^{\circ} 25^{\prime} 35.6^{\prime \prime}$ & 0 & 80 & 20 & $\begin{array}{l}\text { Agricultura } \\
\text { (huertas caseras } \\
\text { con café y } \\
\text { maíz). }\end{array}$ & $\begin{array}{l}\text { Infraestructura humana (zona } \\
\text { suburbana). }\end{array}$ \\
\hline
\end{tabular}


Análisis participativo de servicios ecosistémicos en un área protegida del bosque seco tropical (bs-T), Colombia

Cárdenas-Camacho, L. M., Díaz, S., Gómez-Anaya, W., Rojas-Rojas J. E. y López-Camacho, R.

\begin{tabular}{|c|c|c|c|c|c|c|c|}
\hline 45 & $3^{\circ} 19^{\prime} 06.2^{\prime \prime}$ & $75^{\circ} 19^{\prime} 48.1^{\prime \prime}$ & 0 & 70 & 30 & Ganadería. & Ganadería y quemas. \\
\hline 46 & $3^{\circ} 18^{\prime} 47.6^{\prime \prime}$ & $75^{\circ} 18^{\prime} 31.7^{\prime \prime}$ & 0 & 0 & 100 & $\begin{array}{l}\text { Reforestación } \\
\text { fosfatos del } \\
\text { Huila. Carretera } \\
\text { veredal. }\end{array}$ & $\begin{array}{l}\text { Ganadería e infraestructura } \\
\text { humana (extracción minerales) }\end{array}$ \\
\hline 47 & $3^{\circ} 35^{\prime} 33.9^{\prime \prime}$ & $75^{\circ} 06^{\prime} 54.5^{\prime \prime}$ & 0 & 0 & 100 & \begin{tabular}{|l|} 
Ganadería \\
(bebederos), \\
infraestructura \\
humana (tubería \\
de agua).
\end{tabular} & $\begin{array}{l}\text { Ganadería, infraestructura } \\
\text { humana (carretera Natagai- } \\
\text { ma-Aipe). }\end{array}$ \\
\hline 48 & $3^{\circ} 34^{\prime} 22.1^{\prime \prime}$ & $75^{\circ} 07^{\prime} 34.6^{\prime \prime}$ & 0 & 0 & 100 & Ganadería. & $\begin{array}{l}\text { Ganadería y agricultura (arroz), } \\
\text { Infraestructura humana (carre- } \\
\text { tera divide la unidad). }\end{array}$ \\
\hline 49 & $3^{\circ} 32^{\prime} 52.2^{\prime \prime}$ & $75^{\circ} 07^{\prime} 36.4^{\prime \prime}$ & 0 & 0 & 100 & $\begin{array}{l}\text { Ganadería (be- } \\
\text { bederos). }\end{array}$ & $\begin{array}{l}\text { Ganadería y agricultura (huer- } \\
\text { tas caseras de plátano y yuca). }\end{array}$ \\
\hline 50 & $3^{\circ} 33^{\prime} 33.1^{\prime \prime}$ & $75^{\circ} 06^{\prime} 10.5^{\prime \prime}$ & 0 & 20 & 80 & $\begin{array}{l}\text { Agricultura } \\
\text { (cultivos de } \\
\text { pancoger, } \\
\text { plátano, mango, } \\
\text { naranja, limón), } \\
\text { ganadería. }\end{array}$ & Agricultura (arroz tecnificado). \\
\hline 51 & $3^{\circ} 39^{\prime} 10.7^{\prime \prime}$ & $75^{\circ} 05^{\prime} 19.0^{\prime \prime}$ & 0 & 0 & 100 & $\begin{array}{l}\text { Ganadería (be- } \\
\text { bedero). }\end{array}$ & Ganadería. \\
\hline 52 & $3^{\circ} 47^{\prime} 29.1^{\prime \prime}$ & $75^{\circ} 15^{\prime} 26.0^{\prime \prime}$ & 0 & 0 & 100 & $\begin{array}{l}\text { Ganadería, } \\
\text { agricultura } \\
\text { (plátano). }\end{array}$ & $\begin{array}{l}\text { Ganadería, agricultura (pláta- } \\
\text { no). }\end{array}$ \\
\hline 53 & $3^{\circ} 47^{\prime} 20.1^{\prime \prime}$ & $75^{\circ} 15^{\prime} 18.2^{\prime \prime}$ & 0 & 0 & 100 & $\begin{array}{l}\text { Reserva protec- } \\
\text { tora. } \\
\end{array}$ & $\begin{array}{l}\text { Ganadería y agricultura (huer- } \\
\text { tos caseros: plátano y yuca). }\end{array}$ \\
\hline 54 & $3^{\circ} 44^{\prime} 27.6^{\prime \prime}$ & $75^{\circ} 14^{\prime} 57.8^{\prime \prime}$ & 0 & 0 & 100 & $\begin{array}{l}\text { Ganadería, } \\
\text { infraestructura } \\
\text { humana (vivien- } \\
\text { das y bañadero } \\
\text { regional). }\end{array}$ & $\begin{array}{l}\text { Ganadería y agricultura (huer- } \\
\text { tas caseras: plátano, mango, } \\
\text { mamoncillo). }\end{array}$ \\
\hline 55 & $3^{\circ} 44^{\prime} 27.6^{\prime \prime}$ & $75^{\circ} 14^{\prime} 57.8^{\prime \prime}$ & 0 & 70 & 30 & $\begin{array}{l}\text { Ganadería y } \\
\text { presencia de } \\
\text { basura. }\end{array}$ & $\begin{array}{l}\text { Ganadería e infraestructura } \\
\text { humana (carretera). }\end{array}$ \\
\hline 56 & $3^{\circ} 44^{\prime} 27.6^{\prime \prime}$ & $75^{\circ} 14^{\prime} 57.8^{\prime \prime}$ & 0 & 70 & 0 & Ganadería. & Ganadería. \\
\hline 57 & $3^{\circ} 44^{\prime} 27.6^{\prime \prime}$ & $75^{\circ} 14^{\prime} 57.8^{\prime \prime}$ & & 100 & & Ganadería. & Minifundios, ganadería. \\
\hline 58 & $3^{\circ} 44^{\prime} 27.6^{\prime \prime}$ & $75^{\circ} 14^{\prime} 57.8^{\prime \prime}$ & & 15 & & & $\begin{array}{l}\text { Conjuntos vacacionales y } \\
\text { parcelaciones. }\end{array}$ \\
\hline 59 & $3^{\circ} 44^{\prime} 27.6^{\prime \prime}$ & $75^{\circ} 14^{\prime} 57.8^{\prime \prime}$ & 80 & 20 & & & $\begin{array}{l}\text { Ganadería y agricultura en } \\
\text { pequeña escala. }\end{array}$ \\
\hline 60 & $3^{\circ} 44^{\prime} 27.6^{\prime \prime}$ & $75^{\circ} 14^{\prime} 57.8^{\prime \prime}$ & 5 & 15 & & Ganadería. & $\begin{array}{l}\text { Minifundios con potreros y } \\
\text { cultivos de maíz. }\end{array}$ \\
\hline 61 & $3^{\circ} 44^{\prime} 27.6^{\prime \prime}$ & $75^{\circ} 14^{\prime} 57.8^{\prime \prime}$ & & 10 & & $\begin{array}{l}\text { Cultivos de plá- } \\
\text { tano y cítricos. }\end{array}$ & Ganadería y agricultura. \\
\hline 62 & $3^{\circ} 44^{\prime} 05.0^{\prime \prime}$ & $74^{\circ} 56^{\prime} 00.5^{\prime \prime}$ & 0 & 100 & 0 & $\begin{array}{l}\text { Bebedero, } \\
\text { extracción de } \\
\text { agua. }\end{array}$ & Ganadería. \\
\hline 63 & $3^{\circ} 42^{\prime} 01.0^{\prime \prime}$ & $74^{\circ} 57^{\prime} 18.5^{\prime \prime}$ & 0 & 30 & 0 & $\begin{array}{l}\text { Balneario, } \\
\text { basura. }\end{array}$ & $\begin{array}{l}\text { Ganadería y carretera desta- } \\
\text { pada. }\end{array}$ \\
\hline
\end{tabular}




\begin{tabular}{|c|c|c|c|c|c|c|c|}
\hline 64 & $3^{\circ} 41^{\prime} 38.1^{\prime \prime}$ & $74^{\circ} 57^{\prime} 50.6^{\prime \prime}$ & 0 & 80 & 20 & $\begin{array}{l}\text { Bebedero loca- } \\
\text { lizado. }\end{array}$ & Ganadería. \\
\hline 65 & $3^{\circ} 40^{\prime} 19.8^{\prime \prime}$ & $74^{\circ} 59^{\prime} 15.0^{\prime \prime}$ & 0 & 0 & 100 & Bebederos. & $\begin{array}{l}\text { Huertos caseros. En algunos } \\
\text { sectores plátanos y ganadería }\end{array}$ \\
\hline 66 & $3^{\circ} 39^{\prime} 37.9^{\prime \prime}$ & $75^{\circ} 00^{\prime} 10.6^{\prime \prime}$ & 0 & 40 & 60 & \begin{tabular}{|l|} 
Balneario, \\
extracción de \\
materiales (pie- \\
dra), extracción \\
de agua. \\
\end{tabular} & Ganadería. \\
\hline 67 & $3^{\circ} 39^{\prime} 11.9^{\prime \prime}$ & $75^{\circ} 01^{\prime} 30.2^{\prime \prime}$ & 0 & 40 & 60 & Bebedero. & Ganadería. \\
\hline 68 & $3^{\circ} 33^{\prime} 40.3^{\prime \prime}$ & $75^{\circ} 03^{\prime} 53.4^{\prime \prime}$ & 0 & 40 & 60 & $\begin{array}{l}\text { Bebedero de } \\
\text { ganado. }\end{array}$ & Ganadería. \\
\hline 69 & $3^{\circ} 45^{\prime} 16.4^{\prime \prime}$ & $74^{\circ} 53^{\prime} 11.5^{\prime \prime}$ & 0 & 70 & 30 & $\begin{array}{l}\text { No se eviden- } \\
\text { cia. }\end{array}$ & $\begin{array}{l}\text { Embalse de prado y zona } \\
\text { turística. }\end{array}$ \\
\hline 70 & $3^{\circ} 49^{\prime} 04.4^{\prime \prime}$ & $74^{\circ} 54^{\prime} 57.3^{\prime \prime}$ & 0 & 100 & 0 & \begin{tabular}{|l|} 
Basurero y \\
vertedero aguas \\
negras.
\end{tabular} & Viviendas y huertas caseras. \\
\hline 71 & $3^{\circ} 51^{\prime} 23.1^{\prime \prime}$ & $74^{\circ} 54^{\prime} 10.7^{\prime \prime}$ & 0 & 0 & 100 & Ganadería. & $\begin{array}{l}\text { Ganadería, extracción petrole- } \\
\text { ra, vías. }\end{array}$ \\
\hline 72 & $4^{\circ} 50^{\prime} 21.8^{\prime \prime}$ & $74^{\circ} 44^{\prime} 23.0^{\prime \prime}$ & 0 & 40 & 60 & $\begin{array}{l}\text { Tala selectiva, } \\
\text { cantera. }\end{array}$ & $\begin{array}{l}\text { Infraestructura vial, ganadería, } \\
\text { extracción material. }\end{array}$ \\
\hline 73 & $4^{\circ} 50^{\prime} 01.8^{\prime \prime}$ & $74^{\circ} 38^{\prime} 14.4^{\prime \prime}$ & 50 & 40 & 10 & $\begin{array}{l}\text { Tala selectiva, } \\
\text { caminos. }\end{array}$ & \begin{tabular}{|l|} 
Ganadería doméstica, in- \\
fraestructura vial y doméstica, \\
sísmica, vientos fuertes.
\end{tabular} \\
\hline 74 & $4^{\circ} 50^{\prime} 01.8^{\prime \prime}$ & $74^{\circ} 38^{\prime} 14.4^{\prime \prime}$ & 60 & 40 & 0 & \begin{tabular}{|l|} 
Extracción agua \\
(albercas y \\
mangueras).
\end{tabular} & $\begin{array}{l}\text { Infraestructura vial, agricultura } \\
\text { familiar, ganadería familiar. }\end{array}$ \\
\hline 75 & $4^{\circ} 53^{\prime} 22.4^{\prime \prime}$ & $74^{\circ} 40^{\prime} 33.1^{\prime \prime}$ & 0 & 50 & 50 & \begin{tabular}{|l|} 
Tala carbón \\
vegetal, ganade- \\
ría, tala selecti- \\
va, sísmica.
\end{tabular} & $\begin{array}{l}\text { Ganadería, infraestructura vial } \\
\text { (trituradora), sísmica y quemas. }\end{array}$ \\
\hline 76 & $4^{\circ} 48^{\prime} 48,7^{\prime \prime}$ & $74^{\circ} 43^{\prime} 54.2^{\prime \prime}$ & 10 & 90 & 0 & $\begin{array}{l}\text { Erosión fuerte } \\
\text { ribera del río. } \\
\text { Cárcava, cami- } \\
\text { nos, claros por } \\
\text { caída natural de } \\
\text { árboles. }\end{array}$ & $\begin{array}{l}\text { Ganadería, infraestructura vial, } \\
\text { quemas, spp invasoras (donce- } \\
\text { llo, cuji). }\end{array}$ \\
\hline 77 & $4^{\circ} 45^{\prime} 35,7^{\prime \prime}$ & $74^{\circ} 44^{\prime} 41.0^{\prime \prime}$ & 2 & 18 & 80 & $\begin{array}{l}\text { Tala selectiva, } \\
\text { pastoreo, can- } \\
\text { tera. }\end{array}$ & $\begin{array}{l}\text { Ganadería, infraestructura vial, } \\
\text { fumigación agricultura, petro- } \\
\text { lera (ruido). }\end{array}$ \\
\hline 78 & $4^{\circ} 46^{\prime} 18,5^{\prime \prime}$ & $74^{\circ} 45^{\prime} 45.5^{\prime \prime}$ & 0 & 20 & 80 & Tala selectiva. & $\begin{array}{l}\text { Infraestructura vial, cantera, } \\
\text { cacería. }\end{array}$ \\
\hline 79 & $4^{\circ} 46^{\prime} 45,0^{\prime \prime}$ & $74^{\circ} 45^{\prime} 13.6^{\prime \prime}$ & 0 & 20 & 80 & $\begin{array}{l}\text { Caminos inter- } \\
\text { nos, tala selecti- } \\
\text { va, ramoneo. } \\
\end{array}$ & $\begin{array}{l}\text { Infraestructura vial (avenida } \\
\text { principal). }\end{array}$ \\
\hline 80 & $4^{\circ} 32^{\prime} 07,1^{\prime \prime}$ & $74^{\circ} 46^{\prime} 28.1^{\prime \prime}$ & 20 & 60 & 20 & \begin{tabular}{|l|} 
Caminos, tala \\
selectiva, gana- \\
dería, pequeños \\
cultivos domés- \\
ticos de maíz.
\end{tabular} & $\begin{array}{l}\text { Extracción cantera, infraestruc- } \\
\text { tura vial, ganadería. }\end{array}$ \\
\hline
\end{tabular}




\begin{tabular}{|c|c|c|c|c|c|l|l|}
\hline 81 & $4^{\circ} 32^{\prime} 57,5^{\prime \prime}$ & $74^{\circ} 44^{\prime} 12.8^{\prime \prime}$ & 0 & 70 & 30 & $\begin{array}{l}\text { Ganadería, } \\
\text { extracción de } \\
\text { agua, caminos, } \\
\text { sísmica hace } \\
\text { ocho años cau- } \\
\text { só derrumbe en } \\
\text { nacedero. }\end{array}$ & $\begin{array}{l}\text { Cultivos domésticos, ganade- } \\
\text { ría, infraestructura vial, extrac- } \\
\text { ción material. }\end{array}$ \\
\hline 82 & $4^{\circ} 32^{\prime} 27,4^{\prime \prime}$ & $74^{\circ} 44^{\prime} 04.6^{\prime \prime}$ & 10 & 50 & 40 & $\begin{array}{l}\text { Caminos y } \\
\text { ramoneo. }\end{array}$ & $\begin{array}{l}\text { Infraestructura vial y vivienda. } \\
\text { Agricultura doméstica. }\end{array}$ \\
\hline 83 & $4^{\circ} 36^{\prime} 00,0^{\prime \prime}$ & $74^{\circ} 43^{\prime} 20.7^{\prime \prime}$ & 0 & 100 & 0 & $\begin{array}{l}\text { Ramoneo, } \\
\text { cacería, tala } \\
\text { selectiva, leña } \\
\text { (panela). }\end{array}$ & $\begin{array}{l}\text { Ganadería, sísmica, agricultura } \\
\text { familiar (maíz). }\end{array}$ \\
\hline 84 & $4^{\circ} 27^{\prime} 48,5^{\prime \prime}$ & $74^{\circ} 47^{\prime} 50.6^{\prime \prime}$ & 0 & 20 & 80 & $\begin{array}{l}\text { Caminos, } \\
\text { cercas, vivienda } \\
\text { familia, ramo- } \\
\text { neo doméstico, } \\
\text { erosión media, } \\
\text { claros, sp inva- } \\
\text { soras. }\end{array}$ & $\begin{array}{l}\text { Infraestructura vial, pastos } \\
\text { abandonados, vivienda fami- }\end{array}$ \\
\hline
\end{tabular}

